\section{Fuberidazole (FBZ)}<smiles>c1coc(-c2nc3ccccc3[nH]2)c1</smiles><smiles>c1ccc2[nH]c(-c3cscn3)nc2c1</smiles>

\section{Carbendazim (MBC)}

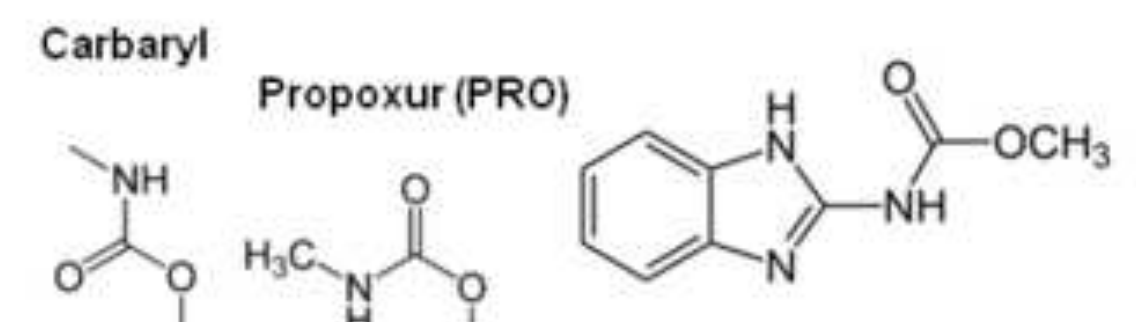<smiles>Cc1ccccc1OC(C)C</smiles>
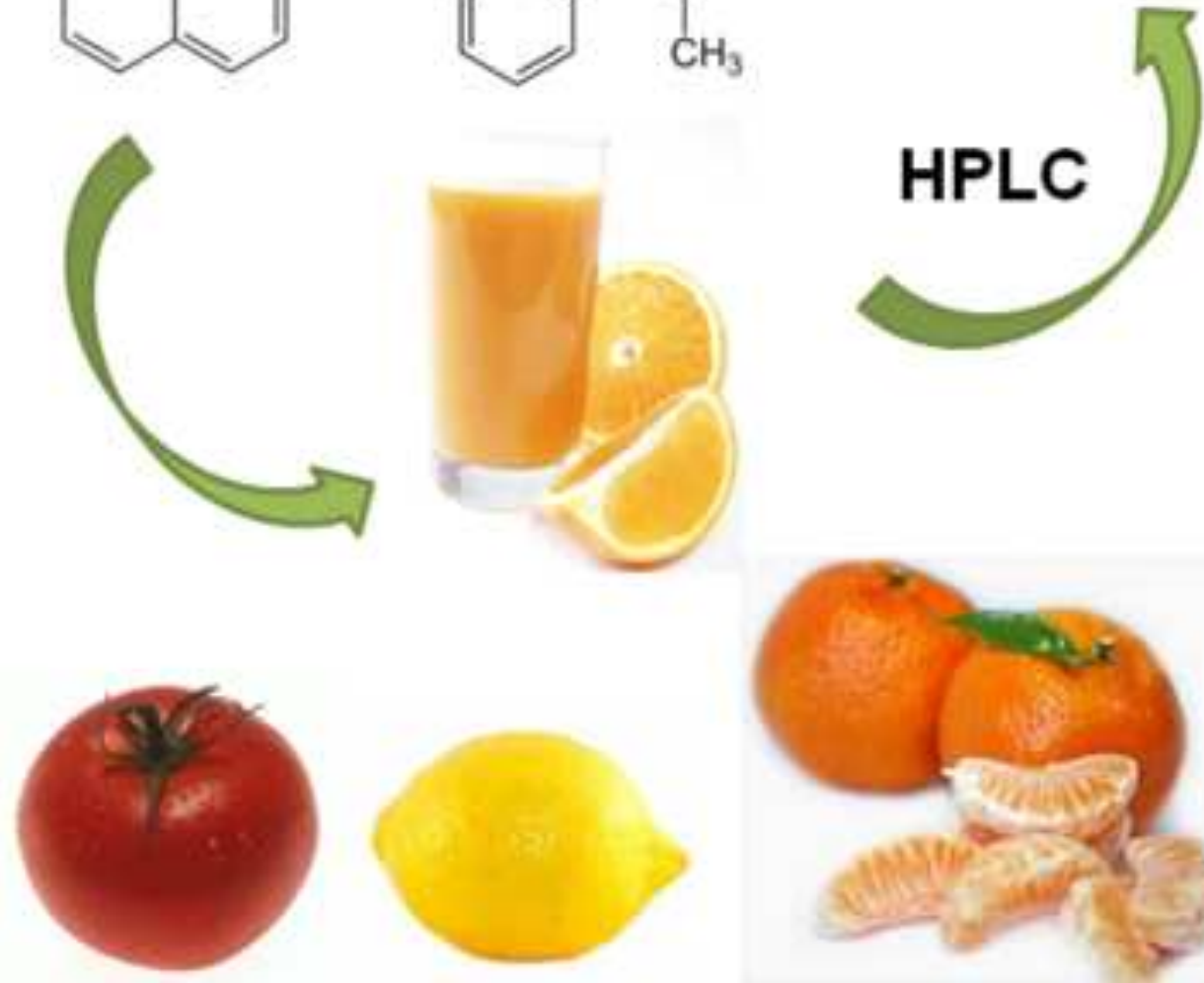

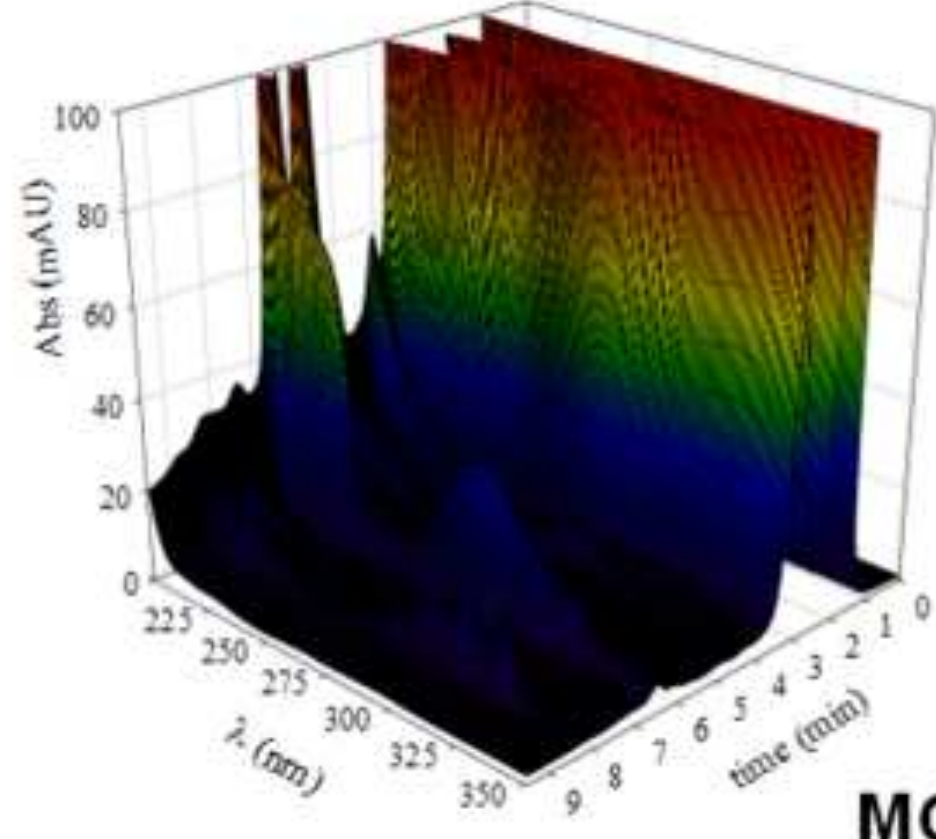

MCR

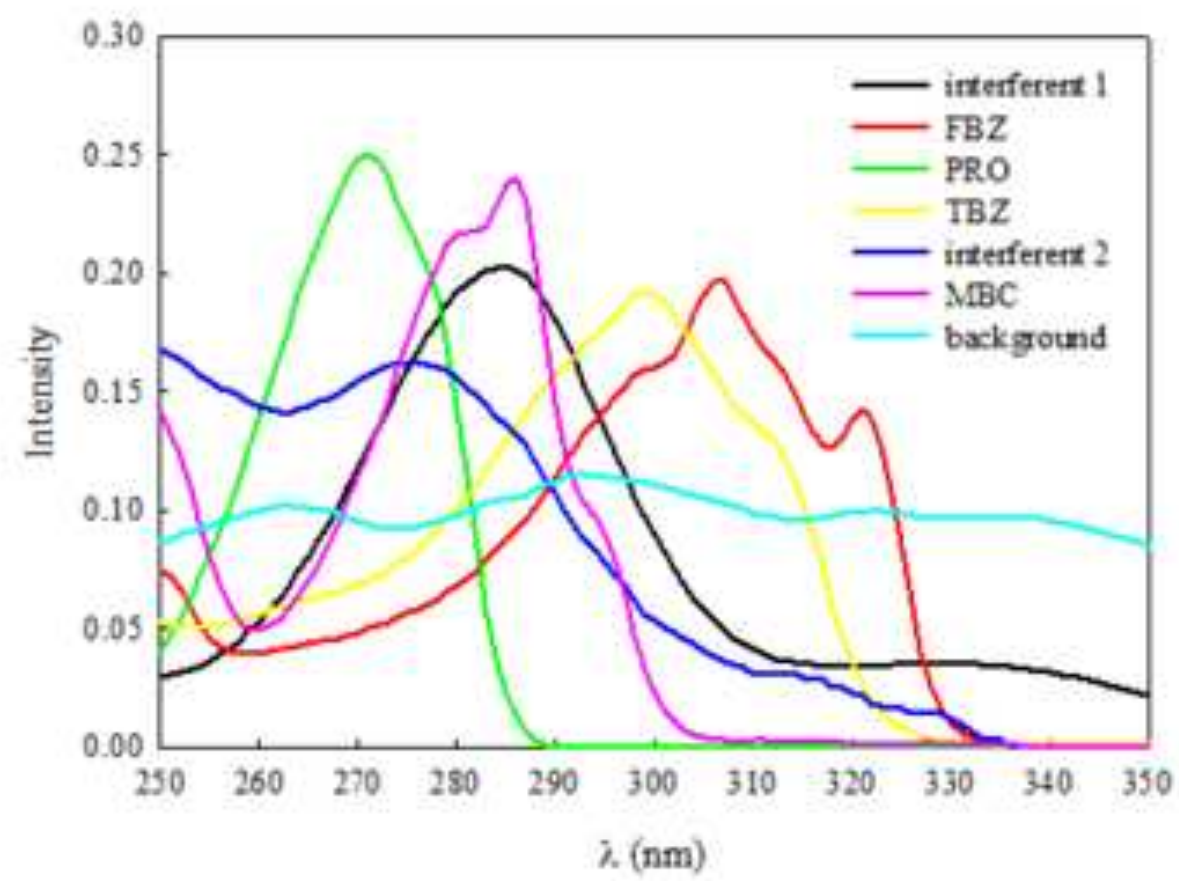


- Five pesticides were determinted in juice, fruit and vegetable samples

- Liquid chromatography was coupled to diode array detection

- Chromatographic-spectral matrices were analyzed by multivariate curve resolution 
1 Determination of five pesticides in juice, fruit and vegetable samples by 2 means of liquid chromatography combined with multivariate curve resolution 3

4 Valeria Boeris, Juan A. Arancibia, Alejandro C. Olivieri

5 Departamento de Química Analítica, Facultad de Ciencias Bioquímicas y

6 Farmacéuticas, Universidad Nacional de Rosario e Instituto de Química Rosario

7 (IQUIR-CONICET), Suipacha 531(S2002LRK) Rosario, Argentina.

8

9 Corresponding author:

10 Alejandro Olivieri

11 Tel./fax: +543414372704 .

12 E-mail addresses: olivieri@iquir-conicet.gov.ar, aolivier@fbioyf.unr.edu.ar 


\section{Abstract}

The aim of this work was to quantify five commonly used pesticides (propoxur, carbaryl, carbendazim, thiabendazole and fuberidazole) in real samples as: tomato, orange juice, grapefruit juice, lemon and tangerine. The method used for the determination of these analytes in the complex matrices was highperformance liquid chromatography with diode array detection. In order to work under isocratic conditions and to complete each run in less than $10 \mathrm{~min}$, the analysis was carried out applying multivariate curve resolution coupled to alternating least-squares (MCR-ALS). The flexibility of this applied multivariate model allowed the prediction of the concentrations of the five analytes in complex samples including strongly coeluting analytes, elution time shifts, band shape changes and presence of uncalibrated interferents. The obtained limits of detection

(in $\mu \mathrm{g} \mathrm{L}^{-1}$ ) using the proposed methodology were 2.3 (carbendazim), 0.90 (thiabendazole), 12 (propoxur), 0.46 (fuberidazole) and 0.32 (carbaryl).

\section{Keywords}

High-performance liquid chromatography; Diode array detection; Multivariate curve resolution; Pesticides; Vegetable samples

\section{Abbreviations}

High-performance liquid chromatography (HPLC), diode array detection (DAD), multivariate curve resolution coupled to alternating least-squares (MCR-ALS), 

37 fuberidazole (FBZ)

38

\section{Introduction}

propoxur (PRO), carbaryl (CBL), carbendazim (MBC), thiabendazole (TBZ),

Although the use of pesticides provides unquestionable benefits in providing a plentiful, low-cost supply of high-quality fruits and vegetables, their incorrect application may leave harmful residues, which involve possible health risk [1]. The concentration of pesticides is regulated in many samples such as drinking waters, vegetables, juices, etc., by the European Commission [2] and the Food and Drug Administration [3], among other agencies. Traditionally, the instrumental techniques employed to determine these compounds involve fluorescence, gas or liquid chromatography [4-8]. Specifically, the determination of benzimidazolic pesticides (carbendazim, thiabendazole and fuberidazole) and/or carbamates (carbaryl, propoxur and carbendazim) in fruits and vegetables have been carried out by various approaches, such as supramolecular solvent-based microextraction followed by high-performance liquid chromatography (HPLC) with fluorescence detection [9], gas chromatography coupled to mass spectrometry and selected ion monitoring [10], enzymatic immunoassay using antibodies [11-13] or electrochemical methods $[14,15]$.

The analysis of mixtures of pesticides using methods based on HPLC sometimes results in complex separations and overlapped peaks [16, 17]. Nevertheless, complex multicomponent mixtures can in many cases be qualitatively and quantitatively resolved by means of chemometrics. Depending on their nature, data can be arranged in a two-way structure (a table or a matrix), as in 
60 the case of collecting the absorbance spectra for many samples, or in a three-way 61 structure, e.g. in HPLC with diode array detection (DAD), where spectra are 62 recorded at several elution times for each sample. Such data arrangements in 63 three- or higher way arrays can be handled using multi-way methods of analysis $64[18,19]$.

Collection of multi-dimensional chromatographic information, and data

66

67

68

69

70

71

72

73

74

75

76

77

78

79

80

81

82 processing by advanced chemometric algorithms constitute a fruitful combination of techniques, recently applied to diverse research areas [20-22]. Chemometrics is required whenever perfect separation of the various sample components cannot be achieved by the employed chromatographic system, leading to overlapping peaks in the elution time mode. In these cases, selectivity may be mathematically restored by applying multivariate data analysis [23]. In particular, the so-called second-order advantage can be achieved, a property which is inherent to matrix instrumental data, and implies that analytes can be quantified in samples containing potential interferences [21]. Signals arising from coeluting analytes or foreign components can be modeled by powerful second-order multivariate algorithms.

The combination of chemometrics to HPLC presents additional advantages in relation to traditional methods: since chemometrics allows resolving coeluted peaks, it is possible to reduce the duration of the chromatographic run, allowing not only processing more samples but also reducing the solvent consumption, saving time and money. Moreover, several authors report that gradient of solvents was required to achieve resolution of the analytes [24-26]: this requirement may be 
avoided using isocratic conditions and resolving the peak by applying chemometrics.

In liquid chromatographic runs, elution time shifts and band shape changes usually occur from sample to sample: in these cases, a useful alternative is to analyze the data with flexible algorithms, which allow a given component to present different time profiles in different samples, such as parallel factor analysis 2 (PARAFAC2) or multivariate curve resolution coupled to alternating least-squares (MCR-ALS) [27]. Recent work from our laboratory indicated better performance with MCR-ALS in the case of multi-analyte quantification in the presence of high overlapping of elution profiles and uncalibrated interferences, mainly because of the possibility of building a more constrained model in MCR-ALS in comparison with PARAFAC2 [22].

In the present report, we selected MCR-ALS as the algorithm of choice for processing HPLC-DAD data, and discuss its behavior towards the quantification of the following five pesticides in fruit and vegetable samples: propoxur (PRO), carbaryl (CBL), carbendazim (MBC), thiabendazole (TBZ) and fuberidazole (FBZ) (Fig. 1). The presence of benzimidazoles, carbamates and their degradation products in waters or food products is potentially harmful for humans due to their proven toxicity. This is the cause of the continued interest in the development of analytical methods for monitoring these families of compounds. Previous chromatographic analysis of the presently studied compounds required up to 35 min $[28,29]$. The aim of this work is to quantify these analytes in complex matrices under HPLC isocratic conditions and in less than $10 \mathrm{~min}$. 
<smiles>CNC(=O)Oc1cccc2ccccc12</smiles><smiles>COC(=O)Nc1nc2ccccc2[nH]1</smiles>

Carbaryl (CBL)<smiles>c1coc(-c2nc3ccccc3[nH]2)c1</smiles>

Fuberidazole (FBZ)<smiles>CNC(=O)Oc1ccccc1OC(C)C</smiles>

Propoxur (PRO)<smiles>c1ccc2[nH]c(-c3cscn3)nc2c1</smiles>

Thiabendazole (TBZ)

\section{2. Theory}

109 The bilinear model assumed by MCR methods is analogous to the 110 generalized Lambert-Beer's law, where the individual responses of each 111 component are additive. In matrix form, this bilinear model is expressed as:

112

$$
\mathbf{D}=\mathbf{C} \mathbf{S}^{\top}+\mathbf{E}
$$

113 where $\mathbf{D}($ size $J \times K)$ is the matrix of experimental data $(J$ is the number of elution

114 time data points and $K$ is the number of absorption wavelengths), $\mathbf{C}$ (size $J \times M$ ) is 115 the matrix whose columns contain the concentration profiles of the $N$ components 116 present in the samples, $\mathbf{S}^{\top}($ size $N \times K)$ is the matrix whose rows contain the 
117 component spectra and $\mathbf{E}\left(\right.$ size $\left.J_{\times} K\right)$ is a matrix collecting the experimental error 118 and the variance not explained by the bilinear model of equation (1).

119 The first step in MCR-ALS studies is to obtain a rough estimation of the 120 number of components, which can be simply performed by visual inspection of 121 singular values or principal component analysis (PCA) [30, 31].

122 The resolution is accomplished using an iterative ALS procedure, initialized 123 using an initial estimation of the spectral or concentration profiles for each 124 intervening species. Different methods are used for this purpose, such as evolving 125 factor analysis [32] or the determination of the purest variables [33]. If the initial 126 estimations are the spectral profiles, the unconstrained least-squares solution for 127 the concentration profiles can be calculated from the expression:

where $\left(\mathbf{S}^{\top}\right)^{+}$is the pseudoinverse of the spectral matrix $\mathbf{S}^{\top}$ [34]. If the initial 130 estimations were the concentration profiles, the unconstrained least-squares 131 solution for the spectra can be calculated from the expression: alternating least-squares cycle, so that, at each iteration, new $\mathbf{C}$ and $\mathbf{S}^{\top}$ matrices

135 are obtained. During these iterative recalculations of $\mathbf{C}$ and $\mathbf{S}^{\top}$, a series of 136 constraints (e.g. non-negativity, unimodality and sample selectivity; the latter 137 removes a component which is known to be absent in a given sample) could be 138 applied to give physical meaning to the obtained solutions, and to limit their 139 possible number for the same data fitting and decrease the extent of possible 
rotation ambiguities [35]. Iterations continue until an optimal solution is obtained that fulfils the postulated constraints and the established convergence criteria.

The procedure described above can be easily extended to the simultaneous analysis of multiple data sets or data matrices if they have at least one data mode (direction) in common. For instance, if the different data sets have been analyzed by the same spectroscopic method, the possible data arrangement and bilinear model extension is given by the following equation:

$$
\mathbf{D}_{\text {aug }}=\left[\begin{array}{c}
\mathbf{D}_{\text {cal1 }} \\
\mathbf{D}_{\text {cal2 }} \\
\ldots \\
\mathbf{D}_{\text {test }}
\end{array}\right]=\left[\begin{array}{c}
\mathbf{C}_{\text {cal1 }} \\
\mathbf{C}_{\text {cal2 }} \\
\ldots \\
\mathbf{C}_{\text {test }}
\end{array}\right] \mathbf{S}^{\mathbf{T}}+\left[\begin{array}{c}
\mathbf{E}_{\text {cal1 }} \\
\mathbf{E}_{\text {cal2 }} \\
\ldots \\
\mathbf{E}_{\text {test }}
\end{array}\right]=\mathbf{C}_{\text {aug }} \mathbf{S}^{\mathbf{T}}+\mathbf{E}_{\text {aug }}
$$

where $\mathbf{D}_{\text {aug }}$ is the augmented data matrix, constructed from I individual data matrices [36], corresponding to the set of calibration samples $\left(\mathbf{D}_{\text {cal1 }}, \mathbf{D}_{\text {cal2 }}, \ldots\right)$ and to a single test sample ( $\left.\boldsymbol{D}_{\text {test }}\right)$.

In this case, $\mathbf{C}_{\text {aug }}$ is the column-wise augmented matrix of concentration profiles (size $J / \times N$, where $N$ is the number of responsive chemical components), $\mathbf{S}^{\top}$ is the matrix of loadings (dimensions $N \times K$ ) in the row vector space, and $\mathbf{E}_{\text {aug }}$ collects the residuals. After decomposition, the scores for analyte $n$ are computed as the sum of the elements of the corresponding profile in each of the sub-matrices of $\mathbf{C}_{\text {aug. }}$

Finally, the calibration scores are employed to build a pseudo-univariate calibration line, leading to an estimation of the corresponding slope $\left(m_{n}\right)$ and offset $\left(n_{n}\right)$. The analyte score in the test sample is then interpolated in the calibration line to yield the predicted analyte concentration $c_{n}$ : 


$$
c_{n}=\left(a_{\text {test }, n}-n_{n}\right) / m_{n}
$$

\section{Experimental}

\subsection{Reagents}

Carbendazim (MBC), thiabendazole (TBZ), fuberidazole (FBZ), propoxur (PRO) and carbaryl (CBL) were purchased from Sigma Aldrich Co. (St. Louis, MO). Methanol was obtained from Merck. Milli-Q water (Millipore) was used in all experiments. Solvents were filtered through $0.45 \mu \mathrm{m}$ filters.

\subsection{Stock standard and working standard solutions}

Stock standard solutions of MBC (570 $\left.\mathrm{mg} \mathrm{L}^{-1}\right)$, TBZ (1150 $\left.\mathrm{mg} \mathrm{L}^{-1}\right)$, FBZ (620

$\left.172 \mathrm{mg} \mathrm{L}{ }^{-1}\right)$, PRO (1720 $\left.\mathrm{mg} \mathrm{L}^{-1}\right)$ and $\mathrm{CBL}\left(680 \mathrm{mg} \mathrm{L}^{-1}\right)$ were prepared in $25.00 \mathrm{~mL}$ 173 volumetric flasks by dissolving accurately weighed amounts of the drugs in 174 methanol and completing to the mark with the same solvent. From these solutions, 175 more diluted solutions were obtained (MBC $22.8 \mathrm{mg} \mathrm{L}^{-1}$, TBZ $20.7 \mathrm{mg} \mathrm{L}^{-1}$, FBZ

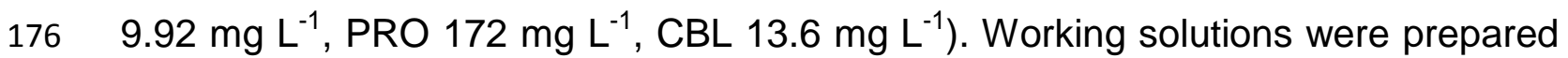
177 immediately before their use by taking appropriate aliquots of solutions and diluting 178 with methanol and water $(50: 50 \mathrm{v} / \mathrm{v})$ to the desired concentrations.

\subsection{Apparatus}

Chromatographic runs were performed on an HP 1200 liquid chromatograph

182 (Agilent Technologies, Waldbronn, Germany) consisting of a quaternary pump, a 183 manual injector fitted with a $200 \mu \mathrm{L}$ loop and a diode array UV-visible detector set 184 at a wavelength range from 200 to $350 \mathrm{~nm}$. A C18 column of $150 \mathrm{~mm} \times 4.6 \mathrm{~mm}, 5 \mu \mathrm{m}$ 
185

186

187

188

189

190

191

192

193

194

195

196

197

198

199

200

201

202

203

204

205

206

207

particle size was employed (Agilent Sorbax SB). The data were collected using the software HP ChemStation for LC Rev.HP 1990-1997.

\subsection{Software}

The data were handled using the MATLAB computer environment [37]. The calculations involved in the mixture resolution by MCR-ALS have been made using mvc2_gui, a MATLAB graphical interface toolbox which is a new version of that already reported in the literature [38].

\subsection{Calibration and validation samples}

In order to design the calibration set, preliminary experiments were performed with the pure analytes, showing that the full elution time range could be divided into three relevant regions: an overlapped zone where three analytes appear (TBZ, PRO and FBZ) and two regions where the remaining two analytes are fully resolved (MBC and $C R L)$. A set of 18 calibration solutions containing the analytes in the ranges 0 - $228 \mu \mathrm{g} \mathrm{L}^{-1}$ for MBC, 0 - $207 \mu \mathrm{g} \mathrm{L}^{-1}$ for TBZ, $0-1720 \mu \mathrm{g} \mathrm{L}$ ${ }^{1}$ for PRO, $0-99.2 \mu \mathrm{g} \mathrm{L}{ }^{-1}$ for FBZ and $0-136 \mu \mathrm{g} \mathrm{L}^{-1}$ for CBL were prepared in appropriate volumetric flasks. The concentrations are collected in Table 1. Fifteen of these samples correspond to the concentrations provided by a central composite design for the three analytes appearing in the overlapped region: TBZ, PRO and FBZ. Each of the remaining three samples of the 18-sample set corresponds to each of the three pure analytes at their maximum levels. Each of these 18 samples was combined with nine equally spaced, duplicate concentration levels for the two 
208

209

210

211

212

213 214 generated within the calibration domain. interval 0-2000 $\mu \mathrm{g} \mathrm{L}^{-1}$.
216

217

218

219

220

221

222

223

224

225

226

227

228

229

resolved analytes. For establishing the calibration concentration ranges, the linear range for all components was studied by analyzing different solutions covering the

A validation set of 10 samples was also prepared, containing the five analytes in concentrations different than those used for calibration, and following a random design, i.e., the specific concentrations were taken as random numbers

\subsection{Samples and sample preparation}

Tangerine, lemon, tomato and commercially available orange and grapefruit juice were purchased from local supermarkets. The fruits and vegetables were chopped into small pieces and processed. Accurately weighted portions of fruits and vegetable samples and aliquots of juice samples were spiked with the assayed pesticides. The semi-solid samples (processed tangerine, lemon and tomato) were blended with water. The $\mathrm{pH}$ of the pesticides-spiked samples was adjusted to neutral by addition of a solution of $\mathrm{NaOH}$. Each sample was centrifuged for 10 min at $4000 \mathrm{~g}$, the supernatant was diluted with methanol and the sample was centrifuged again in the same conditions. Finally, each sample was filtered twice prior to injection: first through a $0.45 \mu \mathrm{m}$ nylon filter and then through a $0.22 \mu \mathrm{m}$ nylon filter.

\subsection{HPLC procedure}


The data matrices were collected using wavelengths from 200 to $350 \mathrm{~nm}$

231 each $1 \mathrm{~nm}$, and each $1.6 \mathrm{~s}$ in the elution time axis. The slit width was $1 \mathrm{~nm}$. The

232 time-absorption matrices were of size $356 \times 151$ and were saved in ASCII format,

233 and transferred to a PC for subsequent manipulation.

234 The mobile phase used for all chromatographic runs was a 50:50 (v/v)

235 mixture of water and methanol, delivered at a flow rate of $1.0 \mathrm{~mL} \mathrm{~min}{ }^{-1}$ with a

236 chromatographic system operating under isocratic mode. Each chromatogram was

237 accomplished in 9.5 minutes.

238

239

\section{Results and discussion}

\subsection{Analysis of the calibration set}

Using pure analyte standards, a chromatographic method allowing their

242 partial separation was developed, making proper selection of the range of detected 243 wavelengths and the composition of the mobile phase, in order to obtain an overall 244 chromatographic time of less than $10 \mathrm{~min}$. Under these conditions, when 245 calibration samples were eluted, a cluster of coeluting peaks and two individual, 246 fully resolved peaks appeared in all chromatographic runs (Fig. 2). Specifically, the 247 MCR-ALS algorithm was used to process LC-DAD matrices taken at specific 248 elution time ranges. Each chromatographic data matrix was divided in the following 249 time regions: region I (3.3-6.9 $\mathrm{min})$ and region II $(7.3-9.5 \mathrm{~min})$. These regions 250 were delimited taking into account the spectrum of each analyte (Fig. 3), i.e., the 251 wavelength ranges required to resolve them. Region I includes the four first eluted 252 analytes: MBC, TBZ, PRO and FBZ. The spectrum of these analytes show that the 253 high sensitivity range is from $250 \mathrm{~nm}$ to $350 \mathrm{~nm}$, thus the wavelength range from 
$254200 \mathrm{~nm}$ to $249 \mathrm{~nm}$ was discarded in their analysis. However, region II includes the 255 last eluted analyte, CBL, whose maximum absorption peak is at $220 \mathrm{~nm}$. In this 256 region, the full wavelength range was selected.

257

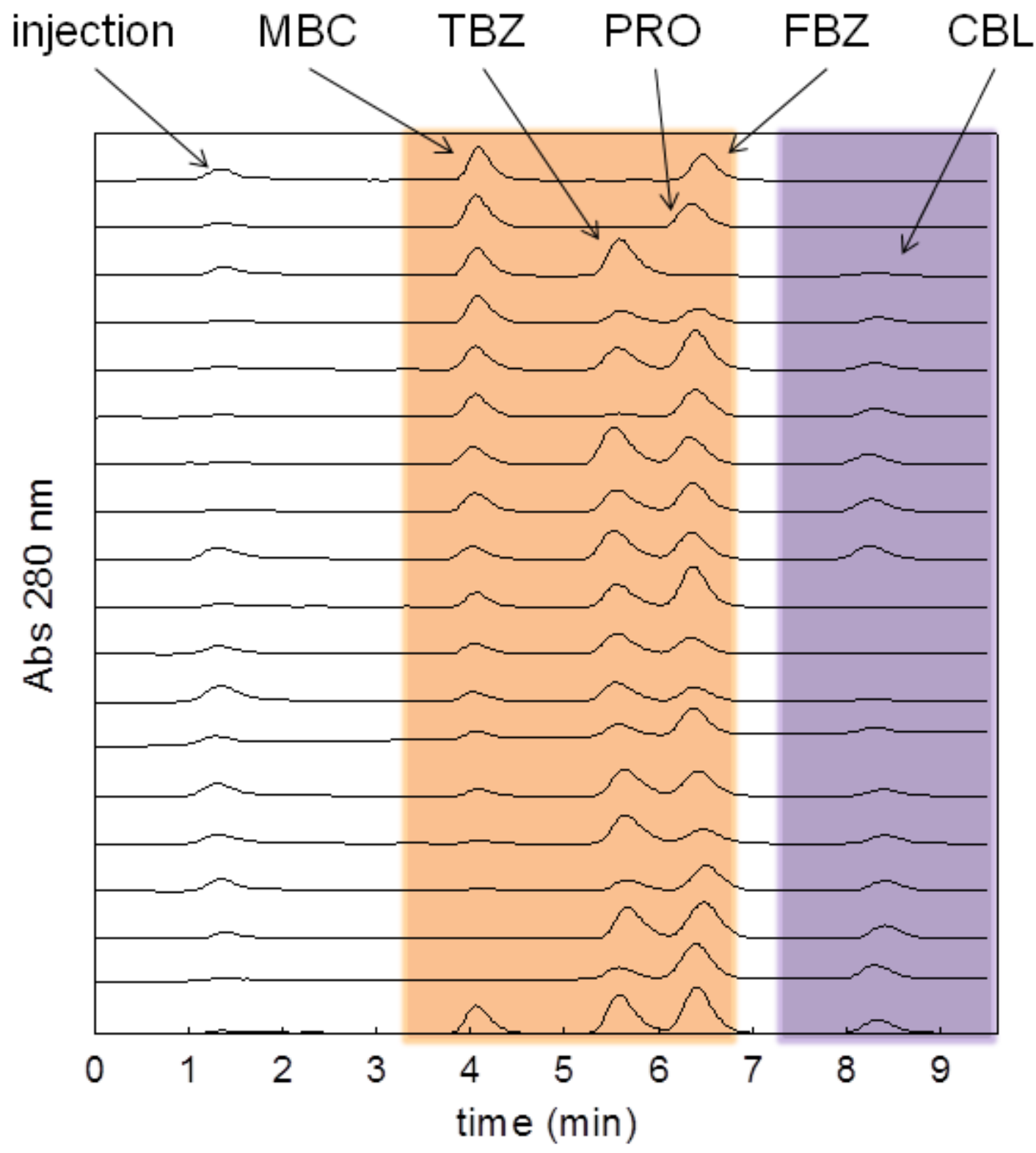

Figure 2 


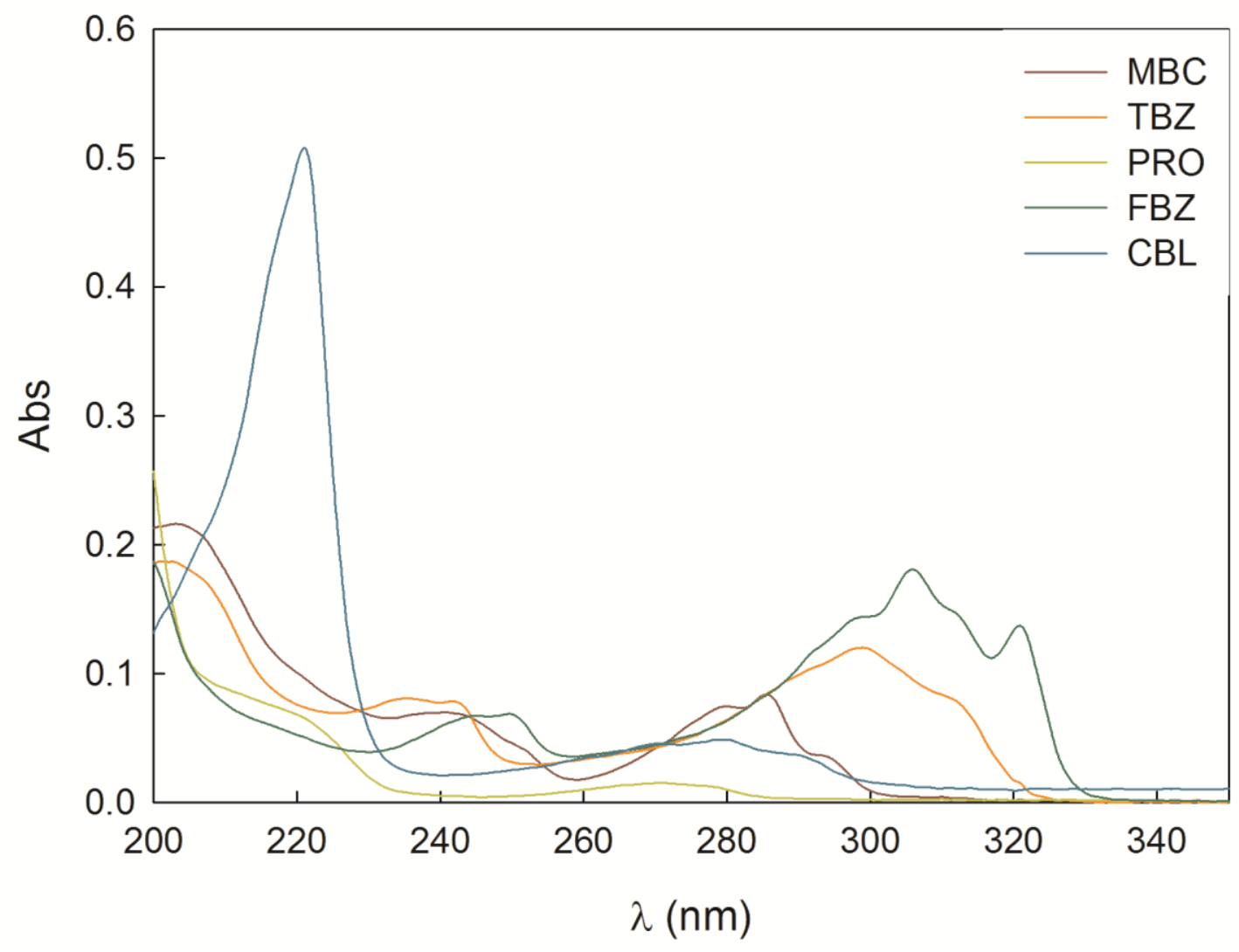

Figure 3

262

$263 \quad$ Notice in Fig. 2 that the the analyte elution time profiles significantly shift 264 from run to run. This effect, combined with the presence of potential interferents in 265 some of the analyzed samples, makes it difficult to align the chromatograms in the 266 time mode, in order to restore the trilinearity required by some second-order 267 multivariate algorithms. This is the main reason for employing the MCR-ALS 268 algorithm for data processing. For each time region, MCR-ALS was applied to 269 augmented matrices in the elution time direction, corresponding to the 270 simultaneous analysis of the HPLC-DAD data matrices for the calibration set of 271 samples. In this analysis, initialization of the multivariate algorithm was performed 
272 using spectral estimates obtained from the analysis of the purest variables. Non-

273 negativity restriction was applied in both modes; unimodality restriction was applied

274 in the elution time mode only to the signals corresponding to the analytes (not to

275 the background signal) but correspondence restriction was not applied during the

276 ALS optimization phase.

277 The number of components was estimated by means of principal component 278 analysis (PCA). The estimated number of components was five in region I and two

279 in region II, which can be justified taking into account the presence of five different 280 signals (corresponding to MBC, TBZ, PRO, FBZ and a background signal) in 281 region I and two different signals (corresponding to $\mathrm{CBL}$ and a background signal) 282 in region II. The resolution of calibration samples provided the characteristic 283 chromatographic profiles and pure spectra for the different analytes plus one signal 284 corresponding to a background. The number of iterations was less than 10 in all 285 cases, with a residual fit lower than $0.07 \mathrm{mUA}$ (region I) and $0.1 \mathrm{mUA}$ (region II). 286 Both residual fits are on the order of the expected instrumental noise associated 287 with DAD detection.

288 After MCR-ALS resolution of the augmented calibration matrix, a pseudo289 univariate calibration was carried out for each compound. The parameters 290 corresponding to the linear regression of the scores from Eq. (5) vs. the 291 corresponding nominal concentrations are shown in Table 2. 

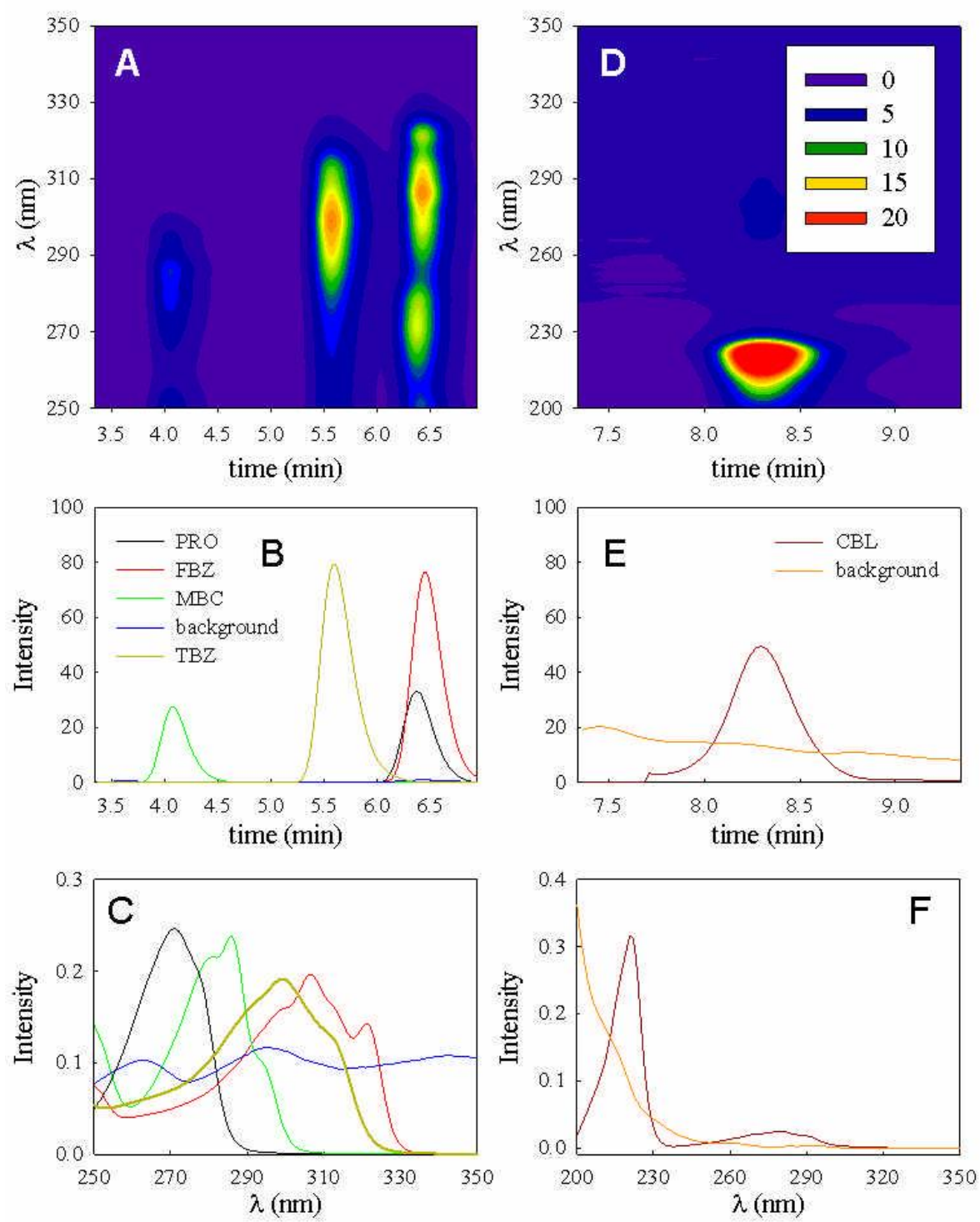

Figure 4

Region I corresponds to the fully overlapped peaks for PRO and FBZ, the 296 partially overlapped peak for TBZ and also to the isolated peak for MBC (Fig. 4A). 297 Five different independent contributions were resolved by MCR-ALS in the first 298 peak cluster, corresponding to region I (Fig. 4A). For a typical sample, the five 299 MCR-ALS resolved elution profiles are shown in Fig. 4B, and the spectra (common 
300 to all samples) in Fig. 4C. These five contributions were identified as the analytes

301 MBC, TBZ, PRO, FBZ and a background signal by comparison of the MCR302 obtained spectra with the actual spectra of the pure compounds (Fig. 3).

303 Coelutions shown in Fig. 4A are untreatable by traditional chromatography; 304 however, mathematical resolution using MCR-ALS was still possible by processing 305 second-order HPLC-DAD data.

306 Region II contained a fully resolved peak at $8.3 \mathrm{~min}$ belonging to $\mathrm{CBL}$. The 307 analysis of $\mathrm{CBL}$ was done both by the traditional method of area measurements 308 and by applying MCR-ALS to the sub-matrix containing its isolated peak. There 309 were not significant differences between the results obtained in both ways $310(p=0.337)$. Figure $4 \mathrm{D}, 4 \mathrm{E}$ and $4 \mathrm{~F}$ show the contour plot, the chromatogram and 311 spectrum corresponding to this region.

\subsection{Analysis of the validation set}

As indicated above, data matrices were analyzed by creating augmented 315 matrices with sub-matrices corresponding to specific time and wavelength windows 316 (regions I and II). For quantifying the analytes in the validation set of samples, each

317 validation HPLC-DAD data matrix was divided into the two selected regions. For 318 each time region, a time mode augmented matrix was created. Each augmented 319 matrix contained, adjacent to each other, the sub-matrices corresponding to the 320 validation samples and to the calibration samples. As before, non-negativity in both 321 modes and unimodality in the time mode (but not correspondence) were applied 322 during ALS optimization. Unimodality was only applied to the signal corresponding 323 to the analytes but not to the background signals. After optimization with the 
324 multivariate algorithm, the scores corresponding to each analyte in each validation

325 sample were isolated, and prediction proceeded by interpolation into the pseudo-

326 univariate score-concentration calibration plot. Linear relationships between MCR-

327 ALS scores and nominal concentrations were found in all cases, supported by the

328 linearity test recommended by IUPAC [39]. The statistical results when MCR-ALS

329 was applied to this validation set are shown in Table 2, implying linearity for all 330 analytes.

As can be observed in Table 3, the predictions for the five analytes are in 332 good agreement with the corresponding nominal values. The root mean square 333 error of prediction (RMSEP) and the relative errors of prediction (REP), computed 334 with respect to the mean calibration concentration of each analyte, can be 335 calculated as follows:

$$
\text { RMSEP }=\sqrt{\frac{\sum_{\mathrm{t}=1}^{\mathrm{T}}\left(\mathrm{y}_{\text {pred }, \mathrm{t}}-\mathrm{y}_{\text {nom } \mathrm{t}}\right)^{2}}{\mathrm{~T}}}
$$

REP $=100 \frac{\text { RMSEP }}{\bar{y}_{\text {cal }}}$

where $y_{\text {pred, } t}$ is the predicted concentration in each sample, $y_{\text {nom, } t}$ is the nominal 339 value of the concentration in the sample, $T$ is he number of test samples, and $\bar{y}_{\text {cal }}$ 340 is the mean calibration concentration. The RMSEP and REP values are also 341 quoted in Table 3. The limits of detection (LOD) and limits of quantification (LOQ) 342 were calculated taking into account the errors of the slope and intercept of the 343 pseudo-univariate calibration curves, as was previously reported by Saurina et al 344 [40]. 


\section{4.3. Analysis of spiked real samples}

Official regulating agencies recommend maximum residue levels (MRL) for

349 the presently studied pesticides which are listed in Table 4 for the assayed fruits

350 and vegetables samples. As can be seen, these values are higher than the

351 calculated LOD (Table 3), and thus analyte pre-concentration is not required.

352 Real fruit and vegetable samples were spiked with these five pesticides and

353 were subjected to the analytical protocol discussed above. The estimated number

354 of components was seven or eight in region I and four in region II, i.e., there are

355 additional components in comparison to the calibration and validation samples.

356 Therefore, the analysis of these samples revealed that there are various interfering

357 species in each region, depending on the sample. 

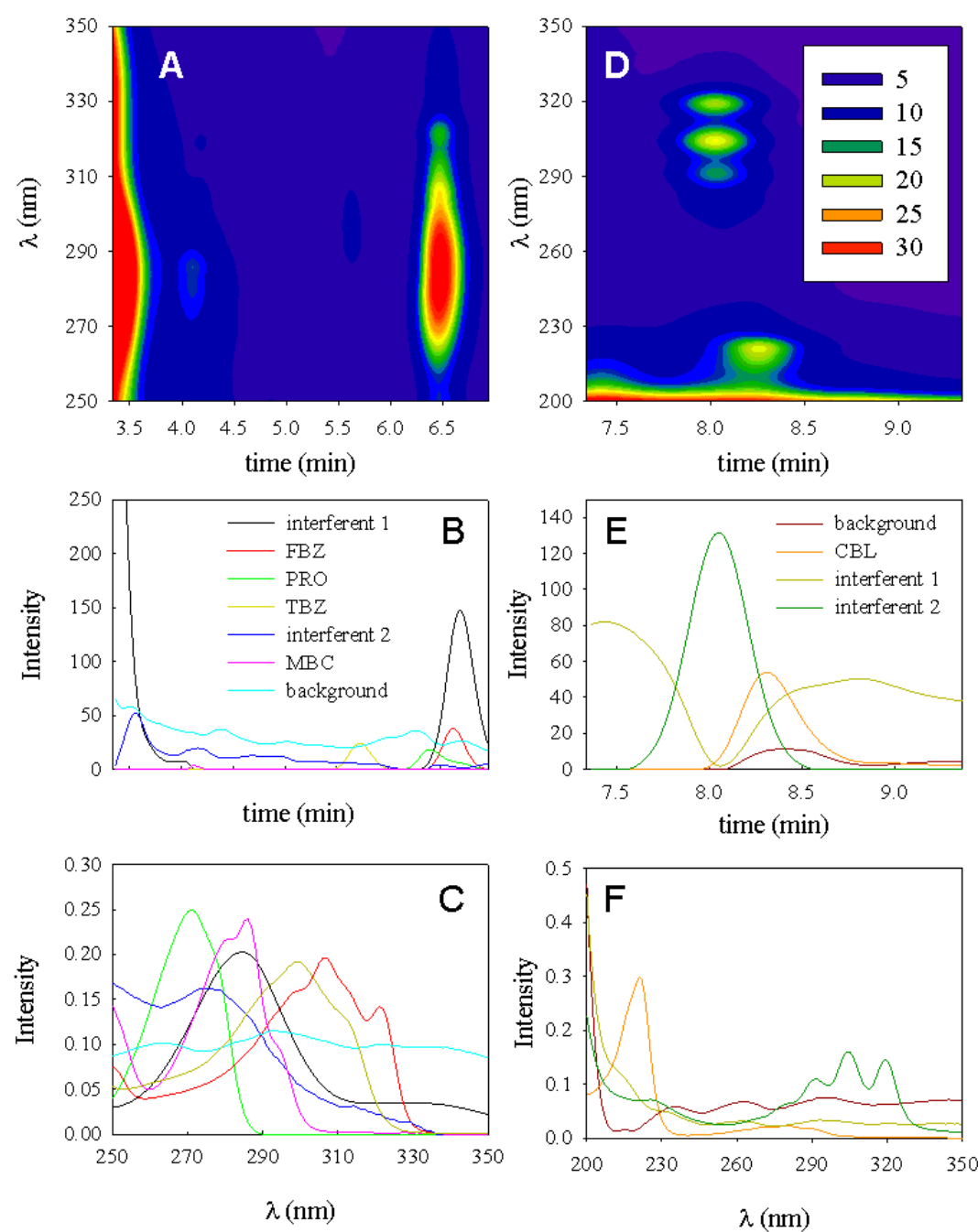

Figure 5

Each data matrix was divided into the two selected regions. As before, non-

361 negativity in both modes and unimodality in the time mode were applied during

362 ALS optimization. Unimodality was only applied to the signal corresponding to the

363 analytes but not to the background signals or to the signals corresponding to

364 interferents. In fact, some of the signals corresponding to interferents have more

365 than one maximum in the time mode. This may be indicating that the interferents

366 are not unique compounds, but also a combination of compounds with similar UV 
spectra that cannot be resolved by MCR. As regards the correspondence

368 restriction (which informs MCR-ALS that the potential interferents are absent in the 369 calibration samples), it is interesting that there was no significant difference when 370 applying correspondence or when this restriction was not applied. The number of 371 iterations was less than 100 in all cases, with a residual fit lower than 0.3 mUA 372 (region I) and $0.45 \mathrm{mUA}$ (region II).

Figure 5 (A to $F)$ shows the contour plot, the chromatogram and spectrum 374 corresponding to both regions for one sample of orange juice. As can be seen, the 375 spectra corresponding to the interfering species were different to those 376 corresponding to the pesticides, allowing their resolution. The recovery results 377 corresponding to different levels of each pesticide the five type of sample assayed 378 are collected in Table 4. As can be appreciated, the predictions for the analytes are 379 in good agreement with the nominal values. If the elliptical joint confidence region 380 is analyzed for the slope and intercept of plot of predicted vs. nominal 381 concentrations we conclude that the ellipse includes the theoretically expected 382 values of $(1,0)$, indicating the accuracy of the used methodology (data not shown). 383 Indeed, a paired t-test indicates no significant difference between the nominal 384 concentrations and the predicted using the presently proposed methodology. The $p$ 385 values are also listed in Table 4. This strongly suggests that HPLC-DAD combined 386 to MCR-ALS is a useful methodology for the analysis of these pesticides in 387 commercial juices, fruit and vegetable samples. 
391

392

393

394

395

396

397

398

399 400 information.

401

\section{Acknowledgements}

403

404

405

406

407

\section{Conclusions}

Complex samples including strongly coeluting analytes, elution time shifts, band shape changes and presence of uncalibrated interferents have been analyzed by HPLC-DAD. The flexibility of the applied multivariate model (MCRALS) allows the prediction of the concentrations of five analytes in a set of validation samples. More importantly, in the most challenging analytical scenario, i.e., real vegetable and fruit samples, these five analytes were quantified within a coeluting cluster in the presence of unwanted and non calibrated signals, achieving the second-order advantage which is inherent to second-order HPLC-DAD

Financial support provided by the University of Rosario, CONICET and ANPCyT (Project No. PIP 2010-0084) is appreciated. Valeria Boeris is grateful to CONICET for her scholarship. 
408

409

410

411

412

413

414

415

416

417

418

419

420

421

422

423

424

425

426

427

428

429

430

431

\section{References}

[1] S. Topuz, G. Özhan, B. Alpertunga, Food Control. 16 (2005), pp. 87-92.

[2] European Commission (http://ec.europa.eu/) Last accessed on 31st October, 2013.

[3] U.S. Department of Heath \& Human Services. U.S. Food and Drug Administration (http://www.fda.gov/) Last accessed on 31st October, 2013.

[4] G.F. Pang, C.L. Fan, Y.M. Liu, Y.Z. Cao, J.J. Zhang, B.L. Fu, X.M. Li, Z.Y. Li, Y.P. Wu, Multi, Food Addit. Contam. 23 (2006), pp. 777-810.

[5] A. Bordagaray, R. Garcia-Arrona, E. Millan, Anal. Methods. 5 (2013), pp. 25652571.

[6] X.Y. Song, Y.P. Shi, J. Chen, Food Chem. 139 (2013), pp. 246-252.

[7] G. Rübensam, F. Barreto, R.B. Hoff, T.M. Pizzolato, Food Control. 29 (2013), pp. 55-60.

[8] C. Carrillo-Carrión, B.M. Simonet, M. Valcárcel, Anal. Chim. Acta. 692 (2011), pp. $103-108$.

[9] A. Moral, M.D. Sicilia, S. Rubio, Anal. Chim. Acta. 650 (2009), pp. 207-213.

[10] G.E. Mercer, J.A. Hurlbut, J. AOAC Int. 87 (2004), pp. 1224-1236.

[11] R.J. Bushway, D.L. Brandon, A.H. Bates, L. Li, K.A. Larkin, B.S. Young, J. Agric. Food. Chem. 43 (1995), pp. 1407-1412.

[12] G.S. Nunes, M.P. Marco, M. Farré, D. Barceló, Anal. Chim. Acta. 387 (1999), pp. 245-253.

[13] J. Sun, T. Dong, Y. Zhang, S. Wang, Anal. Chim. Acta. 666 (2010), pp. 76-82.

[14] G.S. Nunes, D. Barceló, B.S. Grabaric, J.M. Díaz-Cruz, M.L. Ribeiro, Anal. Chim. Acta. 399 (1999), pp. 37-49. 
[15] J. Caetano, S.A.S. Machado, Sens. Actuators, B. 129 (2008), pp. 40-46.

[16] Q. Wu, Q. Chang, C. Wu, H. Rao, X. Zeng, C. Wang, Z. Wang, J. Chromatogr. A. 1217 (2010), pp. 1773-1778.

[17] S. Broecker, F. Pragst, A. Bakdash, S. Herre, M. Tsokos, Forensic Sci. Int., 212 (2011), pp. 215-226.

[18] M.J. Rodríguez-Cuesta, R. Boqué, F.X. Rius, D. Picón Zamora, M. Martínez Galera, A. Garrido Frenich, Anal. Chim. Acta. 491 (2003), pp. 47-56.

[19] M.J. Rodríguez-Cuesta, R. Boqué, F.X. Rius, J.L. Martínez Vidal, A. Garrido Frenich, Chemom. Intell. Lab. Syst. 77 (2005), pp. 251-260.

[20] X.D. Qing, H.L. Wu, C.C. Nie, X.F. Yan, Y.N. Li, J.Y. Wang, R.Q. Yu, Talanta. 103 (2013), pp. 86-94.

[21] R.M. Maggio, P.C. Damiani, A.C. Olivieri, Talanta, 83 (2011), pp. 1173-1180.

[22] S.A. Bortolato, J.A. Arancibia, G.M. Escandar, Anal. Chem. 80 (2008), pp. 8276-8286.

[23] K.S. Booksh, B.R. Kowalski, Anal. Chem. 66 (1994), pp. 782A-791A.

[24] H. Obana, M. Okihashi, K. Akutsu, Y. Kitagawa, S. Hori, J. Agric. Food Chem. 50 (2002), pp. 4464-4467.

[25] S. Seccia, S. Albrizio, P. Fidente, D. Montesano, J. Chromatogr. A. 1218 (2011), pp. 1253-1259.

[26] M. Saraji, N. Tansazan, J. Sep. Sci. 32 (2009), pp. 4186-4192.

[27] Y. Zhou, G. Xu, F.F.K. Choi, L.S. Ding, Q.B. Han, J.Z. Song, C.F. Qiao, Q.S. Zhao, H.X. Xu, J. Chromatogr. A. 1216 (2009), pp. 4847-4858.

[28] A. Belmonte Vega, A. Garrido Frenich, J.L. Martínez Vidal, Anal. Chim. Acta. 538 (2005), pp. 117-127. 
456 [29] M. Fernández, Y. Picó, J. Mañes, J. Chromatogr. A. 871 (2000), pp. 43-56.

457 [30] J. Jaumot, R. Gargallo, A. de Juan, R. Tauler, Chemom. Intell. Lab. Syst. 76 458 (2005), pp. 101-110.

459 [31] M. Maeder, A. Zilian, Chemom. Intell. Lab. Syst. 3 (1988), pp. 205-213.

460 [32] M. Maeder, Anal. Chem. 59 (1987), pp. 527-530.

461 [33] W. Windig, J. Guilment, Anal. Chem. 63 (1991), pp. 1425-1432.

462 [34] G.H. Golub, C.F. Van Loan, Matrix Computations, Johns Hopkins University 463 Press, 1996.

464 [35] R. Tauler, A. Smilde, B. Kowalski, J. Chemom. 9 (1995), pp. 31-58.

465 [36] R. Tauler, M. Maeder, A. de Juan, Multiset Data Analysis: Extended 466 Multivariate Curve Resolution, in: S.D. Brown, R. Tauler, B. Walczak (Eds.) 467 Comprehensive Chemometrics, Elsevier, Oxford, 2009, pp. 473-505.

468 [37] MATLAB version 2011b, The Mathworks Inc., Natick, Massachussets, USA.

469 [38] A.C. Olivieri, H.L. Wu, R.Q. Yu, Chemom. Intell. Lab. Syst. 96 (2009), pp. 246470251.

471 [39] K. Danzer, L.A. Currie, Pure \& Appl. Chem. 70 (1998), pp. 993-1014.

472 [40] J. Saurina, C. Leal, R. Compañó, M. Granados, M.D. Prat, R. Tauler, Anal.

473 Chim. Acta. 432 (2001), pp. 241-251. 
475 Table 1: Calibration concentrations $\left(\mu \mathrm{g} \mathrm{L}^{-1}\right)$ for the five assayed analytes.

\begin{tabular}{|c|c|c|c|c|c|}
\hline Sample & MBC & TBZ & PRO & FBZ & CBL \\
\hline 1 & 0.0 & 62.1 & 1376 & 79.4 & 136.0 \\
\hline 2 & 0.0 & 165.6 & 1376 & 79.4 & 122.4 \\
\hline 3 & 22.8 & 62.1 & 516 & 79.4 & 102.0 \\
\hline 4 & 22.8 & 165.6 & 516 & 29.8 & 81.6 \\
\hline 5 & 57.0 & 165.6 & 1376 & 29.8 & 68.0 \\
\hline 6 & 57.0 & 62.1 & 1376 & 29.8 & 54.4 \\
\hline 7 & 91.2 & 113.8 & 172 & 54.6 & 34.0 \\
\hline 8 & 91.2 & 113.8 & 946 & 9.9 & 13.6 \\
\hline 9 & 114.0 & 113.8 & 1720 & 54.6 & 0.0 \\
\hline 10 & 114.0 & 165.6 & 516 & 79.4 & 136.0 \\
\hline 11 & 136.8 & 113.8 & 946 & 54.6 & 122.4 \\
\hline 12 & 136.8 & 207.0 & 946 & 54.6 & 102.0 \\
\hline 13 & 171.0 & 20.7 & 946 & 54.6 & 81.6 \\
\hline 14 & 171.0 & 113.8 & 946 & 99.2 & 68.0 \\
\hline 15 & 205.2 & 62.1 & 516 & 29.8 & 54.4 \\
\hline 16 & 205.2 & 207.0 & 0 & 0.0 & 34.0 \\
\hline 17 & 228.0 & 0.0 & 1720 & 0.0 & 13.6 \\
\hline 18 & 228.0 & 0.0 & 0 & 99.2 & 0.0 \\
\hline
\end{tabular}

476

477 
478 Table 2: Summary of the results from the pseudo-univariate calibration curves for 479 all analytes $^{\mathrm{a}}$.

\begin{tabular}{|c|c|c|c|c|c|}
\hline & Slope $^{\mathrm{b}}$ & Intercept $^{\mathrm{b}}$ & $r^{2}$ & $s_{\mathrm{y} / \mathrm{x}}$ & $p$ value \\
\hline MBC & $1.48(3)$ & $-2(4)$ & 0.9833 & 14 & 0.161 \\
\hline TBZ & $5.16(7)$ & $11(9)$ & 0.9894 & 29 & 0.464 \\
\hline PRO & $0.252(4)$ & $7(4)$ & 0.9917 & 13 & 0.603 \\
\hline FBZ & $9.8(2)$ & $20(10)$ & 0.9894 & 33 & 0.262 \\
\hline CBL & $12.0(2)$ & $-10(10)$ & 0.9902 & 56 & 0.253 \\
\hline
\end{tabular}

480

$481{ }^{a} r^{2}$, squared correlation coefficient; $s_{y / x}$, standard deviation of regression residuals, $p$ value, 482 probability associated to the IUPAC recommended $F$ test for linearity $(p>0.05$ implies linearity at $48395 \%$ confidence level).

$484{ }^{\mathrm{b}}$ Standard deviation in parenthesis.

485 
Table 3: MCR-ALS results for the prediction of the studied analytes in the validation set of samples.

\begin{tabular}{|c|c|c|c|c|c|c|c|c|c|c|}
\hline \multirow{2}{*}{ Sample } & \multicolumn{2}{|c|}{$\mathrm{MBC}\left(\mu \mathrm{g} \mathrm{L}^{-1}\right)$} & \multicolumn{2}{|c|}{ TBZ $\left(\mu \mathrm{g} \mathrm{L}^{-1}\right)$} & \multicolumn{2}{|c|}{$\mathrm{PRO}\left(\mu \mathrm{g} \mathrm{L}^{-1}\right)$} & \multicolumn{2}{|c|}{$\mathrm{FBZ}\left(\mu \mathrm{g} \mathrm{L}^{-1}\right)$} & \multicolumn{2}{|c|}{$\mathrm{CBL}\left(\mu \mathrm{g} \mathrm{L}^{-1}\right)$} \\
\hline & $\mathrm{N}$ & $\mathrm{P}^{\mathrm{a}}$ & $\bar{N}$ & $\mathrm{P}^{\mathrm{a}}$ & $\bar{N}$ & $\mathrm{P}^{\mathrm{a}}$ & $\bar{N}$ & $\mathrm{P}^{\mathrm{a}}$ & $\mathrm{N}$ & $\mathrm{P}^{\mathrm{a}}$ \\
\hline 1 & 173.0 & $170(6)$ & 95.2 & $87.1(2)$ & 963 & $920(30)$ & 9.9 & $10(2)$ & 12.2 & $11.3(7)$ \\
\hline 2 & 166.0 & $165(4)$ & 137.0 & $136(3)$ & 1030 & $1070(10)$ & 82.3 & $81(4)$ & 121.0 & 123(3) \\
\hline 3 & 0.0 & $1.1(2)$ & 149.0 & $146.7(4)$ & 602 & $570(10)$ & 32.7 & $31(3)$ & 70.7 & $69(2)$ \\
\hline 4 & 228.0 & $234(7)$ & 53.8 & $51(2)$ & 1340 & $1240(60)$ & 21.8 & $21(2)$ & 96.6 & $94(2)$ \\
\hline 5 & 160.0 & $157(5)$ & 164.0 & $158(3)$ & 1170 & $1160(10)$ & 71.4 & $71(4)$ & 132.0 & $125(2)$ \\
\hline 6 & 77.5 & $75.8(4)$ & 51.8 & $54.2(2)$ & 1100 & $1100(10)$ & 36.7 & $36.1(4)$ & 44.9 & $46.1(9)$ \\
\hline 7 & 22.8 & $24(2)$ & 176.0 & $185(2)$ & 1340 & $1360(10)$ & 45.6 & $44.6(4)$ & 46.2 & $47.7(6)$ \\
\hline 8 & 166.0 & $166(5)$ & 74.5 & $73(2)$ & 654 & $603(8)$ & 0.9 & - & 89.8 & $88(2)$ \\
\hline 9 & 185.0 & $188(5)$ & 20.7 & $15.7(1)$ & 1200 & $1180(20)$ & 13.9 & $13(2)$ & 20.4 & $20.5(9)$ \\
\hline 10 & 66.1 & $66(2)$ & 186.0 & $185.8(3)$ & 361 & $340(10)$ & 54.6 & $55(3)$ & 6.8 & $6(2)$ \\
\hline RMSEP & & 6 & & 1.7 & & 43 & & .85 & & $\frac{1}{2.6}$ \\
\hline REP (\%) & & 1 & & .3 & & 4.4 & & 2.3 & & 4.0 \\
\hline LOD & & 3 & & 90 & & 12 & & .46 & & .32 \\
\hline LOQ & & 9 & & 2.7 & & 36 & & 1.4 & & 1.1 \\
\hline Sensitivity & & 92 & & 24 & & 0.018 & & .47 & & 1.2 \\
\hline Selectivity & & 53 & & 29 & & 0.69 & & .31 & & .73 \\
\hline $\begin{array}{l}\text { Analytical } \\
\text { sensitivity }\end{array}$ & & 4 & & 3.7 & & 0.28 & & 7.2 & & 2.9 \\
\hline
\end{tabular}

487

$488 \quad{ }^{a}$ Standard deviation in parenthesis. $\mathrm{N}=$ nominal, $\mathrm{P}=$ predicted. 
Table 4: MCR-ALS results for the prediction of the studied analytes in the spiked samples.

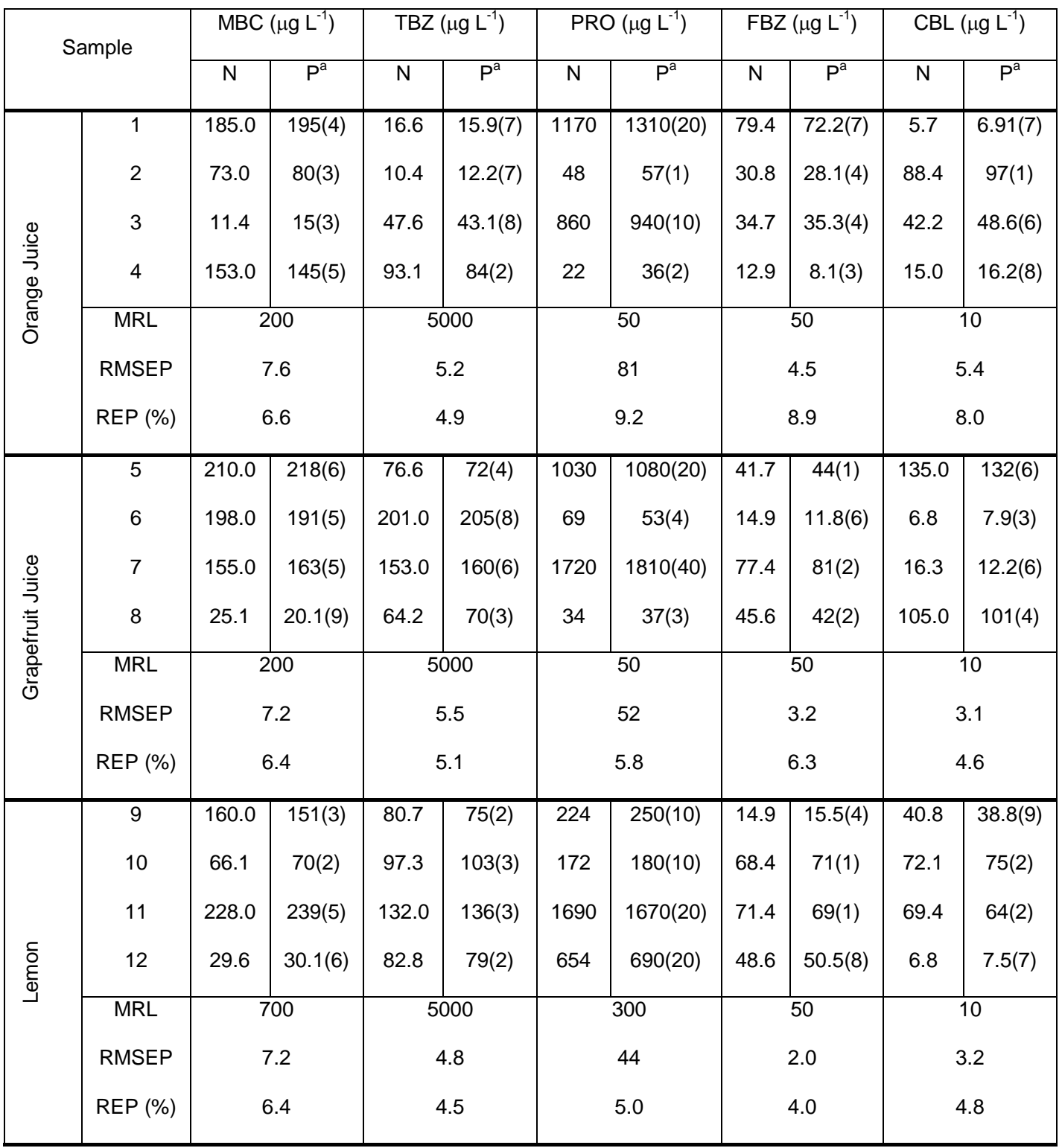

491

This table continues in the next page.

493 
Table 4 (continued)

\begin{tabular}{|c|c|c|c|c|c|c|c|c|c|c|c|}
\hline \multirow{7}{*}{ 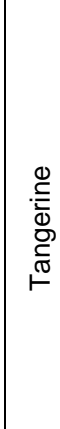 } & 13 & 132.0 & $125(3)$ & 159.0 & $150(10)$ & 1100 & $1070(20)$ & 5.9 & $5.2(1)$ & 132.0 & 125(2) \\
\hline & 14 & 198.0 & $204(5)$ & 97.3 & $102(5)$ & 1200 & $1140(20)$ & 20.8 & 19(1) & 15.0 & 12.1(2) \\
\hline & 15 & 93.5 & $97(2)$ & 207.0 & $200(20)$ & 430 & $440(10)$ & 87.3 & $83(1)$ & 80.2 & $78(1)$ \\
\hline & 16 & 59.3 & 64(2) & 132.0 & $126(6)$ & 155 & $125(7)$ & 41.7 & $47(2)$ & 6.8 & $6.6(3)$ \\
\hline & MRL & \multicolumn{2}{|c|}{$\frac{1}{700}$} & \multicolumn{2}{|c|}{5000} & \multicolumn{2}{|r|}{300} & \multicolumn{2}{|r|}{50} & \multicolumn{2}{|c|}{10} \\
\hline & RMSEP & \multicolumn{2}{|c|}{5.4} & \multicolumn{2}{|c|}{7.1} & \multicolumn{2}{|r|}{39} & \multicolumn{2}{|c|}{3.6} & \multicolumn{2}{|c|}{3.9} \\
\hline & REP (\%) & \multicolumn{2}{|c|}{4.8} & \multicolumn{2}{|c|}{6.7} & \multicolumn{2}{|r|}{4.4} & \multicolumn{2}{|c|}{7.0} & \multicolumn{2}{|c|}{5.8} \\
\hline \multirow{7}{*}{ 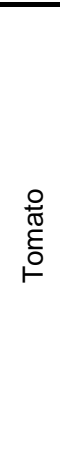 } & $\overline{17}$ & 38.8 & $42(1)$ & 84.9 & $87.6(9)$ & 206 & $166(4)$ & 21.8 & $29.6(4)$ & 124.0 & $131(3)$ \\
\hline & 18 & 80.9 & 76(2) & 97.3 & $91(1)$ & 1010 & $970(10)$ & 25.8 & $23.5(3)$ & 8.2 & $9.8(2)$ \\
\hline & 19 & 108.0 & $115(2)$ & 128.0 & 121(1) & 740 & $797(8)$ & 60.5 & $56.9(7)$ & 59.8 & 65(2) \\
\hline & 20 & 213.0 & 203(3) & 15.5 & $21.3(7)$ & 17 & $38(7)$ & 40.0 & $43.6(6)$ & 24.5 & $21(2)$ \\
\hline & MRL & \multicolumn{2}{|c|}{300} & \multicolumn{2}{|c|}{50} & \multicolumn{2}{|r|}{50} & \multicolumn{2}{|c|}{50} & \multicolumn{2}{|c|}{10} \\
\hline & RMSEP & \multicolumn{2}{|c|}{6.8} & \multicolumn{2}{|c|}{5.8} & & 43 & \multicolumn{2}{|c|}{4.8} & \multicolumn{2}{|c|}{4.8} \\
\hline & REP (\%) & \multicolumn{2}{|c|}{5.9} & \multicolumn{2}{|c|}{5.4} & & 4.9 & \multicolumn{2}{|c|}{9.5} & \multicolumn{2}{|c|}{7.1} \\
\hline & alue & \multicolumn{2}{|c|}{0.389} & \multicolumn{2}{|c|}{0.206} & \multicolumn{2}{|r|}{0.794} & \multicolumn{2}{|c|}{0.439} & \multicolumn{2}{|c|}{0.694} \\
\hline
\end{tabular}

495

$496{ }^{a}$ Standard deviation in parenthesis. $\mathrm{N}=$ nominal, $\mathrm{P}=$ predicted.

497 
499 Figure 1: Chemical structures of the five assayed pesticides.

500

501 Figure 2: Liquid chromatograms ( $\lambda$ of detection: $280 \mathrm{~nm}$ ) for the set of calibration 502 samples. The signal corresponding to each analyte was identified. The subregions 503 selected are highlighted.

504

505 Figure 3: Spectra of pure standards of the five assayed pesticides in medium 506 methanol-water (50:50 v/v). Pesticide concentration: $1 \mathrm{mg} / \mathrm{L}$.

507

508 Figure 4: Results for the analysis of a calibration sample. (A) Surface plot around 509 the first cluster peak (region I) containing the analytes MBC, TBZ, PRO and FBZ 510 (B) MCR-ALS resolved elution profiles for the same sample, with all analytes 511 indicated. (C) Spectral profiles retrieved by MCR-ALS analysis, which are common

512 to all samples. (D) Surface plot around the region II containing CBL (E) MCR-ALS 513 resolved elution profiles in region II. (F) Spectral profiles retrieved by MCR-ALS 514 analysis.

515

516 Figure 5: Results for the analysis of an orange juice sample. (A) Surface plot 517 around the first cluster peak (region I) containing the analytes MBC, TBZ, PRO and 518 FBZ (B) MCR-ALS resolved elution profiles for the same sample, with all analytes 519 indicated. (C) Spectral profiles retrieved by MCR-ALS analysis. (D) Surface plot 
520 around the region II containing CBL (E) MCR-ALS resolved elution profiles in 521 region II. (F) Spectral profiles retrieved by MCR-ALS analysis.

522 
1 Determination of five pesticides in juice, fruit and vegetable samples by 2 means of liquid chromatography combined with multivariate curve resolution 3

4 Valeria Boeris, Juan A. Arancibia, Alejandro C. Olivieri

5 Departamento de Química Analítica, Facultad de Ciencias Bioquímicas y

6 Farmacéuticas, Universidad Nacional de Rosario e Instituto de Química Rosario

7 (IQUIR-CONICET), Suipacha 531(S2002LRK) Rosario, Argentina.

8

9 Corresponding author:

10 Alejandro Olivieri

11 Tel./fax: +543414372704 .

12 E-mail addresses: olivieri@iquir-conicet.gov.ar, aolivier@fbioyf.unr.edu.ar 


\section{Abstract}

The aim of this work was to quantify five commonly used pesticides (propoxur, carbaryl, carbendazim, thiabendazole and fuberidazole) in real samples as: tomato, orange juice, grapefruit juice, lemon and tangerine. The method used for the determination of these analytes in the complex matrices was highperformance liquid chromatography with diode array detection. In order to work under isocratic conditions and to complete each run in less than $10 \mathrm{~min}$, the analysis was carried out applying multivariate curve resolution coupled to alternating least-squares (MCR-ALS). The flexibility of this applied multivariate model allowed the prediction of the concentrations of the five analytes in complex samples including strongly coeluting analytes, elution time shifts, band shape changes and presence of uncalibrated interferents. The obtained limits of detection

(in $\mu \mathrm{g} \mathrm{L}^{-1}$ ) using the proposed methodology were 2.3 (carbendazim), 0.90 (thiabendazole), 12 (propoxur), 0.46 (fuberidazole) and 0.32 (carbaryl).

\section{Keywords}

High-performance liquid chromatography; Diode array detection; Multivariate curve resolution; Pesticides; Vegetable samples

\section{Abbreviations}

High-performance liquid chromatography (HPLC), diode array detection (DAD), multivariate curve resolution coupled to alternating least-squares (MCR-ALS), 

37 fuberidazole (FBZ)

38

\section{Introduction}

propoxur (PRO), carbaryl (CBL), carbendazim (MBC), thiabendazole (TBZ),

Although the use of pesticides provides unquestionable benefits in providing a plentiful, low-cost supply of high-quality fruits and vegetables, their incorrect application may leave harmful residues, which involve possible health risk [1]. The concentration of pesticides is regulated in many samples such as drinking waters, vegetables, juices, etc., by the European Commission [2] and the Food and Drug Administration [3], among other agencies. Traditionally, the instrumental techniques employed to determine these compounds involve fluorescence, gas or liquid chromatography [4-8]. Specifically, the determination of benzimidazolic pesticides (carbendazim, thiabendazole and fuberidazole) and/or carbamates (carbaryl, propoxur and carbendazim) in fruits and vegetables have been carried out by various approaches, such as supramolecular solvent-based microextraction followed by high-performance liquid chromatography (HPLC) with fluorescence detection [9], gas chromatography coupled to mass spectrometry and selected ion monitoring [10], enzymatic immunoassay using antibodies [11-13] or electrochemical methods $[14,15]$.

The analysis of mixtures of pesticides using methods based on highperformance liquid chromatography (HPLC) sometimes results in complex separations and overlapped peaks [16, 17]. Nevertheless, complex multicomponent mixtures can in many cases be qualitatively and quantitatively resolved by means of chemometrics. Depending on their nature, data can be 

61 the absorbance spectra for many samples, or in a three-way structure, e.g. in

arranged in a two-way structure (a table or a matrix), as in the case of collecting HPLC with diode array detection (DAD), where spectra are recorded at several elution times for each sample. Such data arrangements in three- or higher way arrays can be handled using multi-way methods of analysis [18, 19].

Collection of multi-dimensional chromatographic information, and data processing by advanced chemometric algorithms constitute a fruitful combination of techniques, recently applied to diverse research areas [20-22]. Chemometrics is required whenever perfect separation of the various sample components cannot be achieved by the employed chromatographic system, leading to overlapping peaks in the elution time mode. In these cases, selectivity may be mathematically restored by applying multivariate data analysis [23]. In particular, the so-called second-order advantage can be achieved, a property which is inherent to matrix instrumental data, and implies that analytes can be quantified in samples containing potential interferences [21]. Signals arising from coeluting analytes or foreign components can be modeled by powerful second-order multivariate algorithms.

The combination of chemometrics to HPLC presents additional advantages in relation to traditional methods: since chemometrics allows resolving coeluted peaks, it is possible to reduce the duration of the chromatographic run, allowing not only processing more samples but also reducing the solvent consumption, saving time and money. Moreover, several authors report that gradient of solvents was required to achieve resolution of the analytes [24-26]: this requirement may be 
avoided using isocratic conditions and resolving the peak by applying chemometrics.

In liquid chromatographic runs, elution time shifts and band shape changes usually occur from sample to sample: in these cases, a useful alternative is to analyze the data with flexible algorithms, which allow a given component to present different time profiles in different samples, such as parallel factor analysis 2 (PARAFAC2) or multivariate curve resolution coupled to alternating least-squares (MCR-ALS) [27]. Recent work from our laboratory indicated better performance with MCR-ALS in the case of multi-analyte quantification in the presence of high overlapping of elution profiles and uncalibrated interferences, mainly because of the possibility of building a more constrained model in MCR-ALS in comparison with PARAFAC2 [22].

In the present report, we selected MCR-ALS as the algorithm of choice for processing HPLC-DAD data, and discuss its behavior towards the quantification of the following five pesticides in fruit and vegetable samples: propoxur (PRO), carbaryl (CBL), carbendazim (MBC), thiabendazole (TBZ) and fuberidazole (FBZ) (Fig. 1). The presence of benzimidazoles, carbamates and their degradation products in waters or food products is potentially harmful for humans due to their proven toxicity. This is the cause of the continued interest in the development of analytical methods for monitoring these families of compounds. Previous chromatographic analysis of the presently studied compounds required up to 35 min $[28,29]$. The aim of this work is to quantify these analytes in complex matrices under HPLC isocratic conditions and in less than $10 \mathrm{~min}$. 
<smiles>CNC(=O)Oc1cccc2ccccc12</smiles><smiles>COC(=O)Nc1nc2ccccc2[nH]1</smiles>

Carbaryl (CBL)<smiles>c1coc(-c2nc3ccccc3[nH]2)c1</smiles>

Fuberidazole (FBZ)<smiles>CNC(=O)Oc1ccccc1OC(C)C</smiles>

Propoxur (PRO)<smiles>c1ccc2[nH]c(-c3cscn3)nc2c1</smiles>

Thiabendazole (TBZ)

\section{2. Theory}

109 The bilinear model assumed by MCR methods is analogous to the 110 generalized Lambert-Beer's law, where the individual responses of each 111 component are additive. In matrix form, this bilinear model is expressed as:

112

$$
\mathbf{D}=\mathbf{C} \mathbf{S}^{\top}+\mathbf{E}
$$

113 where $\mathbf{D}($ size $J \times K)$ is the matrix of experimental data $(J$ is the number of elution

114 time data points and $K$ is the number of absorption wavelengths), $\mathbf{C}$ (size $J \times M$ ) is 115 the matrix whose columns contain the concentration profiles of the $N$ components 116 present in the samples, $\mathbf{S}^{\top}($ size $N \times K)$ is the matrix whose rows contain the 
117 component spectra and $\mathbf{E}\left(\right.$ size $\left.J_{\times} K\right)$ is a matrix collecting the experimental error 118 and the variance not explained by the bilinear model of equation (1).

119 The first step in MCR-ALS studies is to obtain a rough estimation of the 120 number of components, which can be simply performed by visual inspection of

121 singular values or principal component analysis (PCA) [30, 31]. plots for the matrix

122 of experimental data. This initial number of components can be then refined if 123 necessary, i.e., increasing or decreasing the number of components, depending on 124 their fit and chemical reasonability.

125 The resolution is accomplished using an iterative ALS procedure, initialized 126 using an initial estimation of the spectral or concentration profiles for each 127 intervening species. Different methods are used for this purpose, such as evolving 128 factor analysis [32] or the determination of the purest variables [33]. If the initial 129 estimations are the spectral profiles, the unconstrained least-squares solution for 130 the concentration profiles can be calculated from the expression:

$$
\mathbf{C}=\mathbf{D}\left(\mathbf{S}^{\top}\right)^{+}
$$

132 where $\left(\mathbf{S}^{\top}\right)^{+}$is the pseudoinverse of the spectral matrix $\mathbf{S}_{-}^{\top}$, which is equal to $133\left[\mathbf{S}\left(\boldsymbol{S}^{\top} \mathbf{S}\right)^{-1}\right]$ when $\mathbf{S}^{\mp}$ is full rank [34]. If the initial estimations were the concentration 134 profiles, the unconstrained least-squares solution for the spectra can be calculated 135 from the expression:

$$
\mathbf{S}^{\top}=\mathbf{C}^{+} \mathbf{D}
$$
where $\mathbf{C}^{+}$is the pseudoinverse of $\mathbf{C}\left[\mathbf{C}^{+}=\left(\mathbf{C}^{\top} \mathbf{C}\right)^{-1} \mathbf{C}^{\top}\right]$, when $\mathbf{C}$ is full rank. 138 Both steps can be implemented in an alternating least-squares cycle, so that, at 139 each iteration, new $\mathbf{C}$ and $\mathbf{S}^{\top}$ matrices are obtained. During these iterative 
140 recalculations of $\mathbf{C}$ and $\mathbf{S}^{\top}$, a series of constraints are (e.g. non-negativity,

141 unimodality and sample selectivity; the latter removes a component which is known

142 to be absent in a given sample) could be applied to give physical meaning to the

143 obtained solutions, and to limit their possible number for the same data fitting and

144 decrease the extent of possible rotation ambiguities [35]. Iterations continue until

145 an optimal solution is obtained that fulfils the postulated constraints and the

146 established convergence criteria. Non-negativity constraints may be applied to the

147 concentration profiles, due to the fact that the concentrations of the chemical

148 species are always positive values or zero. Non-negativity constraints can also

149 applied for UV-Vis spectra. Unimodality is a constraint which can be applied to

150 profiles having a single maximum, as in the case of chromatographic profiles.

151 The procedure described above can be easily extended to the simultaneous

152 analysis of multiple data sets or data matrices if they have at least one data mode

153 (direction) in common. For instance, if the different data sets have been analyzed

154 by the same spectroscopic method, the possible data arrangement and bilinear

155 model extension is given by the following equation:

156

$$
\mathbf{D}_{\text {aug }}=\left[\begin{array}{c}
\mathbf{D}_{\text {cal1 }} \\
\mathbf{D}_{\text {cal2 }} \\
\ldots \\
\mathbf{D}_{\text {test }}
\end{array}\right]=\left[\begin{array}{c}
\mathbf{C}_{\text {cal1 }} \\
\mathbf{C}_{\text {cal2 }} \\
\ldots \\
\mathbf{C}_{\text {test }}
\end{array}\right] \mathbf{S}^{\mathrm{T}}+\left[\begin{array}{c}
\mathbf{E}_{\text {cal1 }} \\
\mathbf{E}_{\text {cal2 }} \\
\ldots \\
\mathbf{E}_{\text {test }}
\end{array}\right]=\mathbf{C}_{\text {aug }} \mathbf{S}^{\mathbf{T}}+\mathbf{E}_{\mathrm{aug}}
$$

157 where $\mathbf{D}_{\text {aug }}$ is the augmented data matrix, constructed from I individual data 158 matrices [36], corresponding to the set of calibration samples $\left(\mathbf{D}_{\text {cal1 }}, \mathbf{D}_{\text {cal2 }}, \ldots\right)$ and to 159 a single test sample $\left(\mathbf{D}_{\text {test }}\right)$. Each of these data matrices has size $J \times K$, where $J$ is 160 the number of rows and $K$ is the number of columns. In this column-wise 
161 augmentation mode, the data matrices are placed on top of each other, giving the

162 matrix $\mathbf{D}_{\text {aug }}$ of size $W_{x} K$, which keeps the same number of columns in all of them,

163 and where the different data matrices share their column vector space, $G_{a u g}$ is the

164 column-wise augmented matrix of size $W_{*} N$, and $E_{\text {aug }}$ is the corresponding

165 augmented error matrix. This extended MCR-ALS approach can be used to obtain

166 quantitative determination of an analyte in the presence of other sample

167 components (e.g. interferents).

168 In this case, $\mathbf{C}_{\text {aug }}$ is the column-wise augmented matrix of concentration

169 profiles (size $J / \times N$, where $N$ is the number of responsive chemical components), $\mathbf{S}^{\top}$

170 is the matrix of loadings (dimensions $N \times K$ ) in the row vector space, and $\mathbf{E}_{\text {aug }}$

171 collects the residuals. After decomposition, the scores for analyte $n$ are computed

172 as the sum of the elements of the corresponding profile in each of the sub-matrices

173 of $\mathbf{C}_{\text {aug. }}$ Specifically, the analyte calibration score in the calibration sample $i\left(a_{\text {cali,n }}\right)$

174 is calculated from the elements of the $\boldsymbol{C}_{\text {cali }}$ matrix, which corresponds to the analyte

175 in each calibration sample:

176

$a_{\text {cali }, n}=\sum_{j=1}^{J} c_{\text {cali }}(j, n)$

177 where cali identifies the calibration sample, $n$ the component of interest, jeach of

178 the data points or channels in the sub-matrix along the non-augmented mode and

$179 \epsilon_{c a l i}(j, n)$ the element of the $G_{\text {cali }}$ matrix at channel $j$ for component $n$. On the other

180 hand, the analyte score in the test sample $\left(a_{t e s t, n}\right)$ is defined analogously from the

$181 G_{\text {test }}$ matrix, which corresponds to the analyte in the test sample:

182

$$
a_{\mathrm{test}, n}=\sum_{j=1}^{J} \epsilon_{\mathrm{test}}(j, n)
$$


202

203

204

205

206

where $G_{\text {test }}(j, n)$ is an element of the $C_{\text {test }}$ matrix [see equation (4)].

Finally, the calibration scores are employed to build a pseudo-univariate calibration line, leading to an estimation of the corresponding slope $\left(m_{n}\right)$ and offset $\left(n_{n}\right)$. The analyte score in the test sample is then interpolated in the calibration line to yield the predicted analyte concentration $c_{n}$ :

$$
\begin{aligned}
& c_{n}=\left(a_{\text {test }, n}-n_{n}\right) / m_{n} \\
& \text { In extended MCR-ALS analysis, another useful constraint which can be }
\end{aligned}
$$

applied is the so-called correspondence or sample selectivity, which informs the algorithm that certain components are absent in some samples, e.g., potential interferents may be present in the unknowns but absent in the calibration samples.

\section{Experimental}

\subsection{Reagents}

Carbendazim (MBC), thiabendazole (TBZ), fuberidazole (FBZ), propoxur (PRO) and carbaryl (CBL) were purchased from Sigma Aldrich Co. (St. Louis, MO). Methanol was obtained from Merck. Milli-Q water (Millipore) was used in all experiments. Solvents were filtered through $0.45 \mu \mathrm{m}$ filters.

\subsection{Stock standard and working standard solutions}

Stock standard solutions of MBC (570 mg L-1), TBZ (1150 mg L-1), FBZ (620 $\left.\mathrm{mg} \mathrm{L}^{-1}\right)$, PRO (1720 $\left.\mathrm{mg} \mathrm{L}^{-1}\right)$ and CBL $\left(680 \mathrm{mg} \mathrm{L}^{-1}\right)$ were prepared in $25.00 \mathrm{~mL}$ volumetric flasks by dissolving accurately weighed amounts of the drugs in methanol and completing to the mark with the same solvent. From these solutions, more diluted solutions were obtained (MBC $22.8 \mathrm{mg} \mathrm{L}^{-1}$, TBZ $20.7 \mathrm{mg} \mathrm{L}^{-1}$, FBZ 
$2079.92 \mathrm{mg} \mathrm{L}^{-1}$, PRO $\left.172 \mathrm{mg} \mathrm{L}^{-1}, \mathrm{CBL} 13.6 \mathrm{mg} \mathrm{L}^{-1}\right)$. Working solutions were prepared 208 immediately before their use by taking appropriate aliquots of solutions and diluting 209 with methanol and water $(50: 50 \mathrm{v} / \mathrm{v})$ to the desired concentrations.

\subsection{Apparatus}

Chromatographic runs were performed on an HP 1200 liquid chromatograph (Agilent Technologies, Waldbronn, Germany) consisting of a quaternary pump, a manual injector fitted with a $200 \mu \mathrm{L}$ loop and a diode array UV-visible detector set

215 at a wavelength range from 200 to $350 \mathrm{~nm}$. A C18 column of $150 \mathrm{~mm} \times 4.6 \mathrm{~mm}, 5 \mu \mathrm{m}$ 216 particle size was employed (Agilent Sorbax SB). The data were collected using the 217 software HP ChemStation for LC Rev.HP 1990-1997.

\subsection{Software}

The data were handled using the MATLAB computer environment [37]. The

221 calculations involved in the mixture resolution by MCR-ALS have been made using 222 mvc2_gui, a MATLAB graphical interface toolbox which is a new version of that 223 already reported in the literature [38].

\subsection{Calibration and validation samples}

In order to design the calibration set, preliminary experiments were 227 performed with the pure analytes, showing that the full elution time range could be 228 divided into three relevant regions: an overlapped zone where three analytes 229 appear (TBZ, PRO and FBZ) and two regions where the remaining two analytes 230 are fully resolved (MBC and $\mathrm{CRL}$ ). A set of 18 calibration solutions containing the 
231 analytes in the ranges $0-228 \mu \mathrm{g} \mathrm{L}^{-1}$ for MBC, $0-207 \mu \mathrm{g} \mathrm{L^{-1 }}$ for TBZ, $0-1720 \mu \mathrm{g} \mathrm{L}^{-}$

$232{ }^{1}$ for PRO, 0 - $99.2 \mu \mathrm{g} \mathrm{L}^{-1}$ for FBZ and $0-136 \mu \mathrm{g} \mathrm{L}^{-1}$ for CBL were prepared in 233 appropriate volumetric flasks. The concentrations are collected in Table 1. Fifteen 234 of these samples correspond to the concentrations provided by a central composite 235 design for the three analytes appearing in the overlapped region: TBZ, PRO and 236 FBZ. Each of the remaining three samples of the 18-sample set corresponds to 237 each of the three pure analytes at their maximum levels. Each of these 18 samples 238 was combined with nine equally spaced, duplicate concentration levels for the two 239 resolved analytes. For establishing the calibration concentration ranges, the linear 240 range for all components was studied by analyzing different solutions covering the 241 interval 0-2000 $\mu \mathrm{g} \mathrm{L}^{-1}$.

242 A validation set of 10 samples was also prepared, containing the five 243 analytes in concentrations different than those used for calibration, and following a 244 random design, i.e., the specific concentrations were taken as random numbers 245 generated within the calibration domain.

246

247 3.6. Samples and sample preparation

248 Tangerine, lemon, tomato and commercially available orange and grapefruit 249 juice were purchased from local supermarkets. The fruits and vegetables were 250 chopped into small pieces and processed. Accurately weighted portions of fruits 251 and vegetable samples and aliquots of juice samples were spiked with the assayed 252 pesticides. The semi-solid samples (processed tangerine, lemon and tomato) were 253 blended with water. The $\mathrm{pH}$ of the pesticides-spiked samples was adjusted to 
254 neutral by addition of a solution of $\mathrm{NaOH}$. Each sample was centrifuged for 10 min

255 at $4000 \mathrm{~g}$, the supernatant was diluted with methanol and the sample was 256 centrifuged again in the same conditions. Finally, each sample was filtered twice 257 prior to injection: first through a $0.45 \mu \mathrm{m}$ nylon filter and then through a $0.22 \mu \mathrm{m}$ 258 nylon filter.

259

260

261

262 each $1 \mathrm{~nm}$, and each $1.6 \mathrm{~s}$ in the elution time axis. The slit width was $1 \mathrm{~nm}$. The

263 time-absorption matrices were of size $356 \times 151$ and were saved in ASCll format, 264 and transferred to a PC for subsequent manipulation.

265

The mobile phase used for all chromatographic runs was a 50:50 (v/v)

266 mixture of water and methanol, delivered at a flow rate of $1.0 \mathrm{~mL} \mathrm{~min}{ }^{-1}$ with a 267 chromatographic system operating under isocratic mode. Each chromatogram was 268 accomplished in 9.5 minutes.

269

270

\section{Results and discussion}

\section{4.1. Analysis of the calibration set}

272

Using pure analyte standards, a chromatographic method allowing their

273 partial separation was developed, making proper selection of the range of detected

274 wavelengths and the composition of the mobile phase, in order to obtain an overall

275 chromatographic time of less than $10 \mathrm{~min}$. Under these conditions, when 276 calibration samples were eluted, a cluster of coeluting peaks and two individual, 
277 fully resolved peaks appeared in all chromatographic runs (Fig. 2). Specifically, the 278 MCR-ALS algorithm was used to process LC-DAD matrices taken at specific 279 elution time ranges. Each chromatographic data matrix was divided in the following 280 time regions: region I (3.3-6.9 $\mathrm{min})$ and region II $(7.3-9.5 \mathrm{~min})$. These regions 281 were delimited taking into account the spectrum of each analyte (Fig. 3), i.e., the 282 wavelength ranges required to resolve them. Region I includes the four first eluted 283 analytes: MBC, TBZ, PRO and FBZ. The spectrum of these analytes show that the 284 high sensitivity range is from $250 \mathrm{~nm}$ to $350 \mathrm{~nm}$, thus the wavelength range from $285200 \mathrm{~nm}$ to $249 \mathrm{~nm}$ was discarded in their analysis. However, region II includes the 286 last eluted analyte, CBL, whose maximum absorption peak is at $220 \mathrm{~nm}$. In this 287 region, the full wavelength range was selected. 


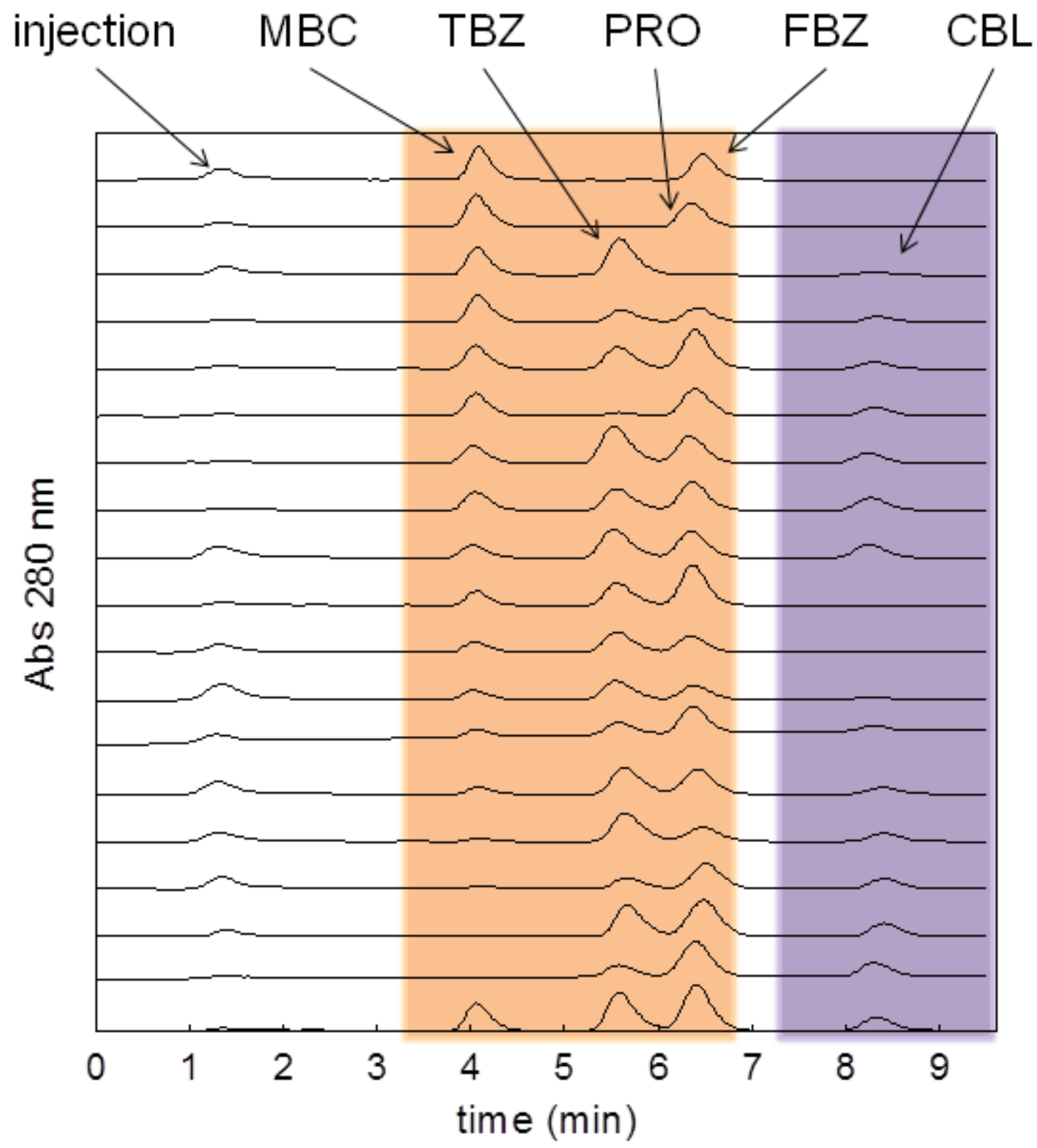

Figure 2 


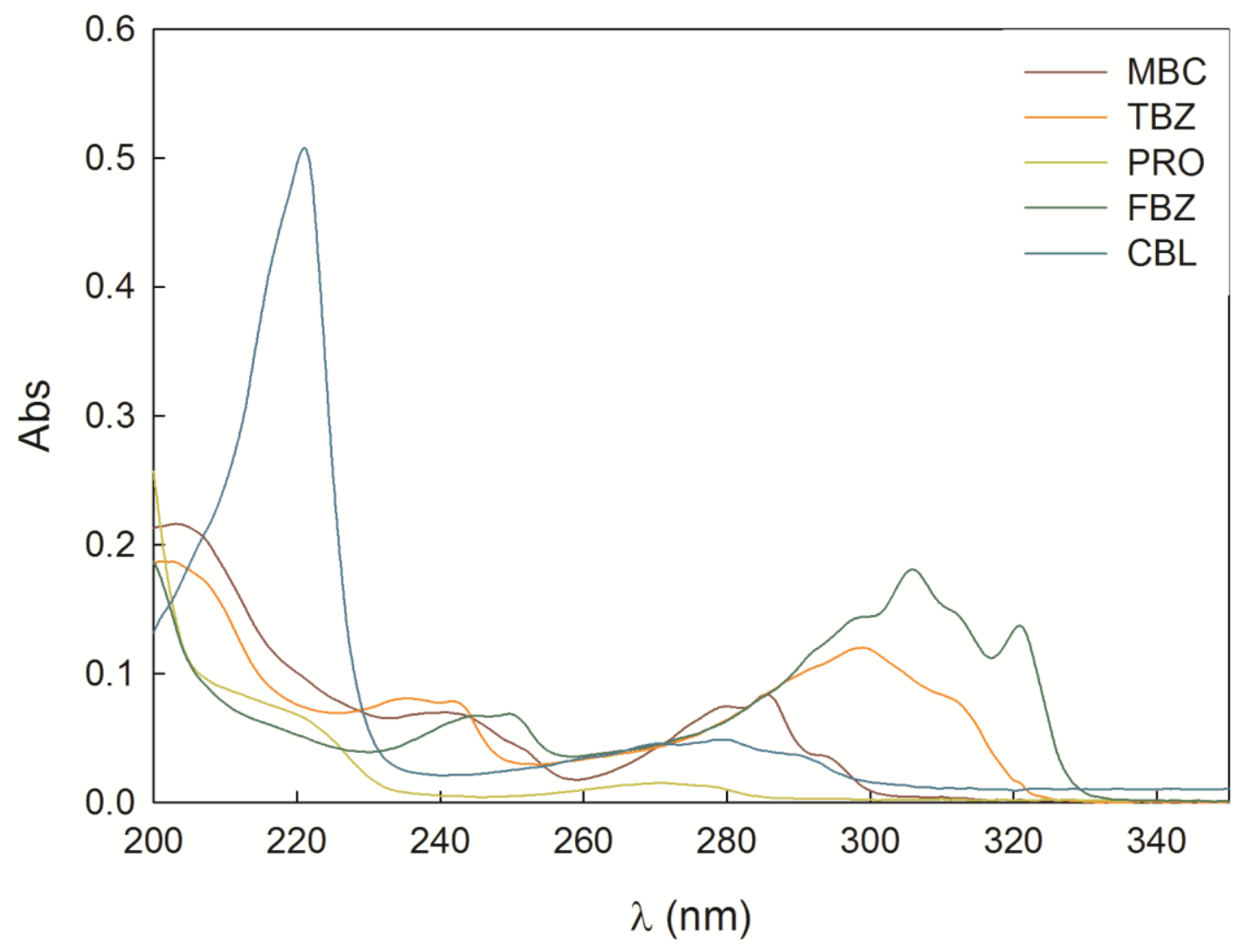

Figure 3

295 from run to run. This effect, combined with the presence of potential interferents in 296 some of the analyzed samples, makes it difficult to align the chromatograms in the 297 time mode, in order to restore the trilinearity required by some second-order 298 multivariate algorithms. This is the main reason for employing the MCR-ALS 299 algorithm for data processing. For each time region, MCR-ALS was applied to 300 augmented matrices in the elution time direction, corresponding to the 301 simultaneous analysis of the HPLC-DAD data matrices for the calibration set of 302 samples. In this analysis, initialization of the multivariate algorithm was performed 
303

304

305

306

307

308

309

310

311

312

313

314

315

316

317

318

319

320

321

322

323

324

325

326

using spectral estimates obtained from the analysis of the purest variables. Nonnegativity restriction was applied in both modes; unimodality restriction was applied in the elution time mode only to the signals corresponding to the analytes (not to the background signal) but correspondence restriction was not applied during the ALS optimization phase.

The number of components was estimated by means of principal component analysis (PCA). PCA is a mathematical procedure that uses orthogonal transformation to convert a set of observations of possibly correlated variables into a set of values of linearly uncorrelated variables called principal components: the first principal component has the largest possible variance (that is, accounts for as much of the variability in the data as possible), and each succeeding component in turn has the highest variance possible under the constraint that it be orthogonal to the preceding components. The estimated number of components was five in region I and two in region II, which can be justified taking into account the presence of five different signals (corresponding to MBC, TBZ, PRO, FBZ and a background signal) in region $\mathrm{I}$ and two different signals (corresponding to $\mathrm{CBL}$ and a background signal) in region II. The resolution of calibration samples provided the characteristic chromatographic profiles and pure spectra for the different analytes plus one signal corresponding to a background. The number of iterations was less than 10 in all cases, with a residual fit lower than 0.07 mUA (region I) and $0.1 \mathrm{mUA}$ (region II). Both residual fits are on the order of the expected instrumental noise associated with DAD detection.

After MCR-ALS resolution of the augmented calibration matrix, a pseudounivariate calibration was carried out for each compound. The parameters 
328 corresponding nominal concentrations are shown in Table 2.
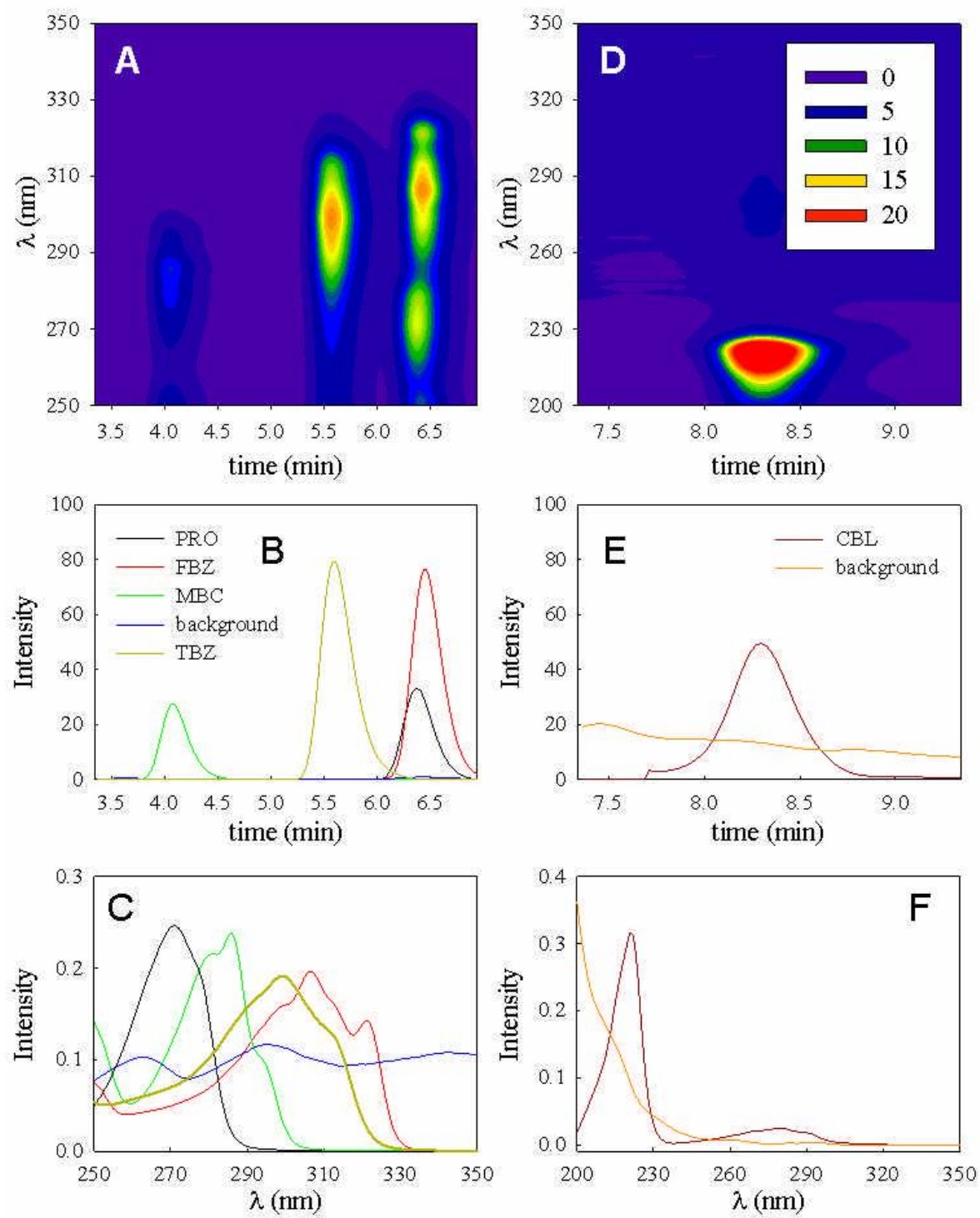

Figure 4

Region I corresponds to the fully overlapped peaks for PRO and FBZ, the 333 partially overlapped peak for TBZ and also to the isolated peak for MBC (Fig. 4A). 334 Five different independent contributions were resolved by MCR-ALS in the first 
peak cluster, corresponding to region I (Fig. 4A). For a typical sample, the five MCR-ALS resolved elution profiles are shown in Fig. 4B, and the spectra (common to all samples) in Fig. 4C. These five contributions were identified as the analytes MBC, TBZ, PRO, FBZ and a background signal by comparison of the MCRobtained spectra with the actual spectra of the pure compounds (Fig. 3). Coelutions shown in Fig. 4A are untreatable by traditional chromatography; however, mathematical resolution using MCR-ALS was still possible by processing second-order HPLC-DAD data.

Region II contained a fully resolved peak at 8.3 min belonging to CBL. The analysis of CBL was done both by the traditional method of area measurements and by applying MCR-ALS to the sub-matrix containing its isolated peak. There were not significant differences between the results obtained in both ways $(p=0.337)$. Figure $4 D, 4 E$ and $4 F$ show the contour plot, the chromatogram and spectrum corresponding to this region.

\subsection{Analysis of the validation set}

As indicated above, data matrices were analyzed by creating augmented matrices with sub-matrices corresponding to specific time and wavelength windows (regions I and II). For quantifying the analytes in the validation set of samples, each validation HPLC-DAD data matrix was divided into the two selected regions. For each time region, a time mode augmented matrix was created. Each augmented matrix contained, adjacent to each other, the sub-matrices corresponding to the validation samples and to the calibration samples. As before, non-negativity in both modes and unimodality in the time mode (but not correspondence) were applied 
during ALS optimization. Unimodality was only applied to the signal corresponding

360 to the analytes but not to the background signals. After optimization with the 361 multivariate algorithm, the scores corresponding to each analyte in each validation

362 sample were isolated, and prediction proceeded by interpolation into the pseudo363 univariate score-concentration calibration plot. Good Linear relationships between

364 MCR-ALS scores and nominal concentrations were found in all cases, supported 365 by the linearity test recommended by IUPAC [39]. The statistical results when 366 MCR-ALS was applied to this validation set are shown in Table 2, implying 367 linearity for all analytes. 370 error of prediction (RMSEP) and the relative errors of prediction (REP), computed 371 with respect to the mean calibration concentration of each analyte, can be 372 calculated as follows:

RMSEP $=\sqrt{\frac{\sum_{\mathrm{t}=1}^{\mathrm{T}}\left(\mathrm{y}_{\text {pred }, \mathrm{t}}-\mathrm{y}_{\text {nom } \mathrm{t}}\right)^{2}}{\mathrm{~T}}}$

$\operatorname{REP}=100 \frac{\text { RMSEP }}{\bar{y}_{\text {cal }}}$

375 where $y_{\text {pred, } t}$ is the predicted concentration in each sample, $y_{\text {nom, } t}$ is the nominal 376 value of the concentration in the sample, $T$ is he number of test samples, and $\bar{y}_{\text {cal }}$ 377 is the mean calibration concentration. The RMSEP and REP values are also 378 quoted in Table 3. The limits of detection (LOD) and limits of quantification (LOQ) 379 were calculated taking into account the errors of the slope and intercept of the 
pseudo-univariate calibration curves, as was previously reported by Saurina et al 381 [40].

382

\subsection{Analysis of spiked real samples}

Official regulating agencies recommend maximum residue levels $(M R L)$ for 385 the presently studied pesticides which are listed in Table 4 for the assayed fruits 386 and vegetables samples. As can be seen, these values are higher than the 387 calculated LOD (Table 3), and thus analyte pre-concentration is not required.

$388 \quad$ Real fruit and vegetable samples were spiked with these five pesticides and 389 were subjected to the analytical protocol discussed above. The estimated number 390 of components was seven or eight in region I and four in region II, i.e., there are 391 additional components in comparison to the calibration and validation samples. 392 Therefore, the analysis of these samples revealed that there are various interfering 393 species in each region, depending on the sample. 

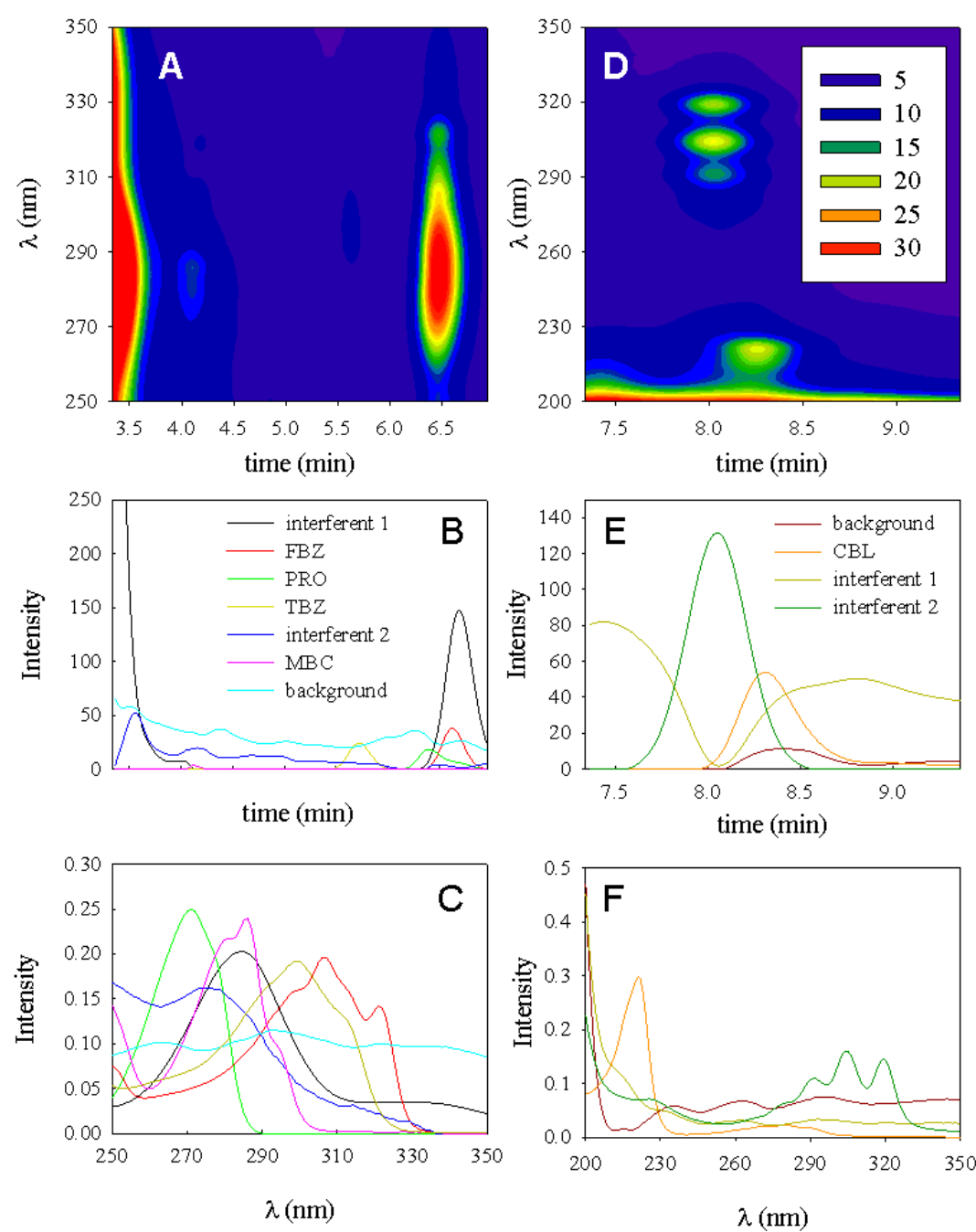

Figure 5

Each data matrix was divided into the two selected regions. As before, non-

397 negativity in both modes and unimodality in the time mode were applied during 398 ALS optimization. Unimodality was only applied to the signal corresponding to the 399 analytes but not to the background signals or to the signals corresponding to 400 interferents. In fact, some of the signals corresponding to interferents have more 401 than one maximum in the time mode. This may be indicating that the interferents 402 are not unique compounds, but also a combination of compounds with similar UV 
spectra that cannot be resolved by MCR. As regards the correspondence

404 restriction (which informs MCR-ALS that the potential interferents are absent in the 405 calibration samples), it is interesting that there was no significant difference when 406 applying correspondence or when this restriction was not applied. The number of 407 iterations was less than 100 in all cases, with a residual fit lower than 0.3 mUA 408 (region I) and 0.45 mUA (region II).

Figure 5 (A to $F)$ shows the contour plot, the chromatogram and spectrum corresponding to both regions for one sample of orange juice. As can be seen, the 411 spectra corresponding to the interfering species were different to those 412 corresponding to the pesticides, allowing their resolution. The recovery results 413 corresponding to different levels of each pesticide the five type of sample assayed 414 are collected in Table 4. As can be appreciated, the predictions for the analytes are 415 in good agreement with the nominal values. If the elliptical joint confidence region 416 is analyzed for the slope and intercept of plot of predicted vs. nominal 417 concentrations we conclude that the ellipse includes the theoretically expected 418 values of $(1,0)$, indicating the accuracy of the used methodology (data not shown). 419 Indeed, a paired t-test indicates no significant difference between the nominal 420 concentrations and the predicted using the presently proposed methodology. The $p$ 421 values are also listed in Table 4. This strongly suggests that HPLC-DAD combined 422 to MCR-ALS is a useful methodology for the analysis of these pesticides in 423 commercial juices, fruit and vegetable samples. 
427

428

429

430

431

432 validation samples. More importantly, in the most challenging analytical scenario,

433 i.e., real vegetable and fruit samples, these five analytes were quantified within a

434 coeluting cluster in the presence of unwanted and non calibrated signals, achieving

435 the second-order advantage which is inherent to second-order HPLC-DAD 436 information.

438 Acknowledgements

Financial support provided by the University of Rosario, CONICET and ANPCyT (Project No. PIP 2010-0084) is appreciated. Valeria Boeris is grateful to 441 CONICET for her scholarship. 


\section{References}

445 [1] S. Topuz, G. Özhan, B. Alpertunga, Food Control. 16 (2005), pp. 87-92.

446 [2] European Commission (http://ec.europa.eu/) Last accessed on 31st October, 4472013.

448 [3] U.S. Department of Heath \& Human Services. U.S. Food and Drug 449 Administration (http://www.fda.gov/) Last accessed on 31st October, 2013.

450 [4] G.F. Pang, C.L. Fan, Y.M. Liu, Y.Z. Cao, J.J. Zhang, B.L. Fu, X.M. Li, Z.Y. Li, 451 Y.P. Wu, Multi, Food Addit. Contam. 23 (2006), pp. 777-810.

452 [5] A. Bordagaray, R. Garcia-Arrona, E. Millan, Anal. Methods. 5 (2013), pp. 25654532571.

454 [6] X.Y. Song, Y.P. Shi, J. Chen, Food Chem. 139 (2013), pp. 246-252.

455 [7] G. Rübensam, F. Barreto, R.B. Hoff, T.M. Pizzolato, Food Control. 29 (2013), 456 pp. 55-60.

457 [8] C. Carrillo-Carrión, B.M. Simonet, M. Valcárcel, Anal. Chim. Acta. 692 (2011), 458 pp. $103-108$

459 [9] A. Moral, M.D. Sicilia, S. Rubio, Anal. Chim. Acta. 650 (2009), pp. 207-213.

460 [10] G.E. Mercer, J.A. Hurlbut, J. AOAC Int. 87 (2004), pp. 1224-1236.

461 [11] R.J. Bushway, D.L. Brandon, A.H. Bates, L. Li, K.A. Larkin, B.S. Young, J. 462 Agric. Food. Chem. 43 (1995), pp. 1407-1412.

463 [12] G.S. Nunes, M.P. Marco, M. Farré, D. Barceló, Anal. Chim. Acta. 387 (1999), 464 pp. 245-253.

465 [13] J. Sun, T. Dong, Y. Zhang, S. Wang, Anal. Chim. Acta. 666 (2010), pp. 76-82.

466 [14] G.S. Nunes, D. Barceló, B.S. Grabaric, J.M. Díaz-Cruz, M.L. Ribeiro, Anal. 467 Chim. Acta. 399 (1999), pp. 37-49. 
468

469

470

471

472

473

474

475

476

477

478

479

480

481

482

483

484

485

486

487

488

489

490

491

[15] J. Caetano, S.A.S. Machado, Sens. Actuators, B. 129 (2008), pp. 40-46.

[16] Q. Wu, Q. Chang, C. Wu, H. Rao, X. Zeng, C. Wang, Z. Wang, J. Chromatogr.

A. 1217 (2010), pp. 1773-1778.

[17] S. Broecker, F. Pragst, A. Bakdash, S. Herre, M. Tsokos, Forensic Sci. Int., 212 (2011), pp. 215-226.

[18] M.J. Rodríguez-Cuesta, R. Boqué, F.X. Rius, D. Picón Zamora, M. Martínez

Galera, A. Garrido Frenich, Anal. Chim. Acta. 491 (2003), pp. 47-56.

[19] M.J. Rodríguez-Cuesta, R. Boqué, F.X. Rius, J.L. Martínez Vidal, A. Garrido

Frenich, Chemom. Intell. Lab. Syst. 77 (2005), pp. 251-260.

[20] X.D. Qing, H.L. Wu, C.C. Nie, X.F. Yan, Y.N. Li, J.Y. Wang, R.Q. Yu, Talanta. 103 (2013), pp. 86-94.

[21] R.M. Maggio, P.C. Damiani, A.C. Olivieri, Talanta, 83 (2011), pp. 1173-1180.

[22] S.A. Bortolato, J.A. Arancibia, G.M. Escandar, Anal. Chem. 80 (2008), pp. 8276-8286.

[23] K.S. Booksh, B.R. Kowalski, Anal. Chem. 66 (1994), pp. 782A-791A.

[24] H. Obana, M. Okihashi, K. Akutsu, Y. Kitagawa, S. Hori, J. Agric. Food Chem. 50 (2002), pp. 4464-4467.

[25] S. Seccia, S. Albrizio, P. Fidente, D. Montesano, J. Chromatogr. A. 1218 (2011), pp. 1253-1259.

[26] M. Saraji, N. Tansazan, J. Sep. Sci. 32 (2009), pp. 4186-4192.

[27] Y. Zhou, G. Xu, F.F.K. Choi, L.S. Ding, Q.B. Han, J.Z. Song, C.F. Qiao, Q.S. Zhao, H.X. Xu, J. Chromatogr. A. 1216 (2009), pp. 4847-4858.

[28] A. Belmonte Vega, A. Garrido Frenich, J.L. Martínez Vidal, Anal. Chim. Acta. 538 (2005), pp. 117-127. 
493 [30] J. Jaumot, R. Gargallo, A. de Juan, R. Tauler, Chemom. Intell. Lab. Syst. 76 494 (2005), pp. 101-110.

495 [31] M. Maeder, A. Zilian, Chemom. Intell. Lab. Syst. 3 (1988), pp. 205-213.

496 [32] M. Maeder, Anal. Chem. 59 (1987), pp. 527-530.

497 [33] W. Windig, J. Guilment, Anal. Chem. 63 (1991), pp. 1425-1432.

498 [34] G.H. Golub, C.F. Van Loan, Matrix Computations, Johns Hopkins University 499 Press, 1996.

500 [35] R. Tauler, A. Smilde, B. Kowalski, J. Chemom. 9 (1995), pp. 31-58.

501 [36] R. Tauler, M. Maeder, A. de Juan, Multiset Data Analysis: Extended 502 Multivariate Curve Resolution, in: S.D. Brown, R. Tauler, B. Walczak (Eds.) 503 Comprehensive Chemometrics, Elsevier, Oxford, 2009, pp. 473-505.

504 [37] MATLAB version 2011b, The Mathworks Inc., Natick, Massachussets, USA.

505 [38] A.C. Olivieri, H.L. Wu, R.Q. Yu, Chemom. Intell. Lab. Syst. 96 (2009), pp. 246506251.

507 [39] K. Danzer, L.A. Currie, Pure \& Appl. Chem. 70 (1998), pp. 993-1014.

508 [40] J. Saurina, C. Leal, R. Compañó, M. Granados, M.D. Prat, R. Tauler, Anal. 509 Chim. Acta. 432 (2001), pp. 241-251. 
511 Table 1: Calibration concentrations $\left(\mu \mathrm{g} \mathrm{L}^{-1}\right)$ for the five assayed analytes.

\begin{tabular}{|c|c|c|c|c|c|}
\hline Sample & MBC & TBZ & PRO & FBZ & CBL \\
\hline 1 & 0.0 & 62.1 & 1376 & 79.4 & 136.0 \\
\hline 2 & 0.0 & 165.6 & 1376 & 79.4 & 122.4 \\
\hline 3 & 22.8 & 62.1 & 516 & 79.4 & 102.0 \\
\hline 4 & 22.8 & 165.6 & 516 & 29.8 & 81.6 \\
\hline 5 & 57.0 & 165.6 & 1376 & 29.8 & 68.0 \\
\hline 6 & 57.0 & 62.1 & 1376 & 29.8 & 54.4 \\
\hline 7 & 91.2 & 113.8 & 172 & 54.6 & 34.0 \\
\hline 8 & 91.2 & 113.8 & 946 & 9.9 & 13.6 \\
\hline 9 & 114.0 & 113.8 & 1720 & 54.6 & 0.0 \\
\hline 10 & 114.0 & 165.6 & 516 & 79.4 & 136.0 \\
\hline 11 & 136.8 & 113.8 & 946 & 54.6 & 122.4 \\
\hline 12 & 136.8 & 207.0 & 946 & 54.6 & 102.0 \\
\hline 13 & 171.0 & 20.7 & 946 & 54.6 & 81.6 \\
\hline 14 & 171.0 & 113.8 & 946 & 99.2 & 68.0 \\
\hline 15 & 205.2 & 62.1 & 516 & 29.8 & 54.4 \\
\hline 16 & 205.2 & 207.0 & 0 & 0.0 & 34.0 \\
\hline 17 & 228.0 & 0.0 & 1720 & 0.0 & 13.6 \\
\hline 18 & 228.0 & 0.0 & 0 & 99.2 & 0.0 \\
\hline
\end{tabular}


514 Table 2: Summary of the results from the pseudo-univariate calibration curves for

515 all analytes $^{\mathrm{a}}$.

\begin{tabular}{|c|c|c|c|c|c|}
\hline & Slope $^{\mathrm{b}}$ & Intercept $^{\mathrm{b}}$ & $r^{2}$ & $s_{\mathrm{y} / \mathrm{x}}$ & $p$ value \\
\hline MBC & $1.48(3)$ & $-2(4)$ & 0.9833 & 14 & 0.161 \\
\hline TBZ & $5.16(7)$ & $11(9)$ & 0.9894 & 29 & 0.464 \\
\hline PRO & $0.252(4)$ & $7(4)$ & 0.9917 & 13 & 0.603 \\
\hline FBZ & $9.8(2)$ & $20(10)$ & 0.9894 & 33 & 0.262 \\
\hline CBL & $12.0(2)$ & $-10(10)$ & 0.9902 & 56 & 0.253 \\
\hline
\end{tabular}

516

$517 a^{a} r^{2}$, squared correlation coefficient; $s_{y / x}$, standard deviation of regression residuals, $p$ value,

518 probability associated to the IUPAC recommended $F$ test for linearity $(p>0.05$ implies linearity at

$51995 \%$ confidence level).

$520{ }^{b}$ Standard deviation in parenthesis.

521 
522 Table 3: MCR-ALS results for the prediction of the studied analytes in the validation set of samples.

\begin{tabular}{|c|c|c|c|c|c|c|c|c|c|c|}
\hline \multirow{2}{*}{ Sample } & \multicolumn{2}{|c|}{$\operatorname{MBC}\left(\mu \mathrm{g} \mathrm{L}^{-1}\right)$} & \multicolumn{2}{|c|}{ TBZ $\left(\mu \mathrm{g} \mathrm{L}^{-1}\right)$} & \multicolumn{2}{|c|}{ PRO $\left(\mu \mathrm{g} \mathrm{L}^{-1}\right)$} & \multicolumn{2}{|c|}{ FBZ $\left(\mu \mathrm{g} \mathrm{L}^{-1}\right)$} & \multicolumn{2}{|c|}{$\mathrm{CBL}\left(\mu \mathrm{g} \mathrm{L}^{-1}\right)$} \\
\hline & $\mathrm{N}$ & $\mathrm{P}^{\mathrm{a}}$ & $\mathrm{N}$ & $\mathrm{P}^{a}$ & $\mathrm{~N}$ & $\mathrm{P}^{\mathrm{a}}$ & $\mathrm{N}$ & $\mathrm{P}^{\mathrm{a}}$ & $\mathrm{N}$ & $\mathrm{P}^{\mathrm{a}}$ \\
\hline 1 & 173.0 & $170(6)$ & 95.2 & $87.1(2)$ & 963 & $920(30)$ & 9.9 & $10(2)$ & 12.2 & $11.3(7)$ \\
\hline 2 & 166.0 & $165(4)$ & 137.0 & $136(3)$ & 1030 & $1070(10)$ & 82.3 & $81(4)$ & 121.0 & $123(3)$ \\
\hline 3 & 0.0 & $1.1(2)$ & 149.0 & $146.7(4)$ & 602 & $570(10)$ & 32.7 & $31(3)$ & 70.7 & $69(2)$ \\
\hline 4 & 228.0 & $234(7)$ & 53.8 & $51(2)$ & 1340 & $1240(60)$ & 21.8 & $21(2)$ & 96.6 & $94(2)$ \\
\hline 5 & 160.0 & $157(5)$ & 164.0 & $158(3)$ & 1170 & $1160(10)$ & 71.4 & $71(4)$ & 132.0 & $125(2)$ \\
\hline 6 & 77.5 & $75.8(4)$ & 51.8 & 54.2(2) & 1100 & $1100(10)$ & 36.7 & $36.1(4)$ & 44.9 & $46.1(9)$ \\
\hline 7 & 22.8 & $24(2)$ & 176.0 & $185(2)$ & 1340 & $1360(10)$ & 45.6 & $44.6(4)$ & 46.2 & $47.7(6)$ \\
\hline 8 & 166.0 & $166(5)$ & 74.5 & $73(2)$ & 654 & $603(8)$ & 0.9 & - & 89.8 & $88(2)$ \\
\hline 9 & 185.0 & $188(5)$ & 20.7 & $15.7(1)$ & 1200 & $1180(20)$ & 13.9 & $13(2)$ & 20.4 & $20.5(9)$ \\
\hline 10 & 66.1 & $66(2)$ & 186.0 & 185.8(3) & 361 & $340(10)$ & 54.6 & $55(3)$ & 6.8 & $6(2)$ \\
\hline RMSEP & & & & 1.7 & & 43 & & 85 & & 6 \\
\hline REP (\%) & & & & .3 & & 4.4 & & .3 & & 0 \\
\hline LOD & & & & 90 & & 12 & & 46 & & 32 \\
\hline LOQ & & & & 2.7 & & 36 & & .4 & & 1 \\
\hline Sensitivity & & & & 24 & & .018 & & 47 & & 2 \\
\hline Selectivity & & & & 29 & & 0.69 & & 31 & & 73 \\
\hline $\begin{array}{l}\text { Analytıcal } \\
\text { sensitivity }\end{array}$ & & & & 3.7 & & 0.28 & & .2 & & 9 \\
\hline
\end{tabular}

523

$524{ }^{a}$ Standard deviation in parenthesis. $\mathrm{N}=$ nominal, $\mathrm{P}=$ predicted.

525 
526 Table 4: MCR-ALS results for the prediction of the studied analytes in the spiked samples.

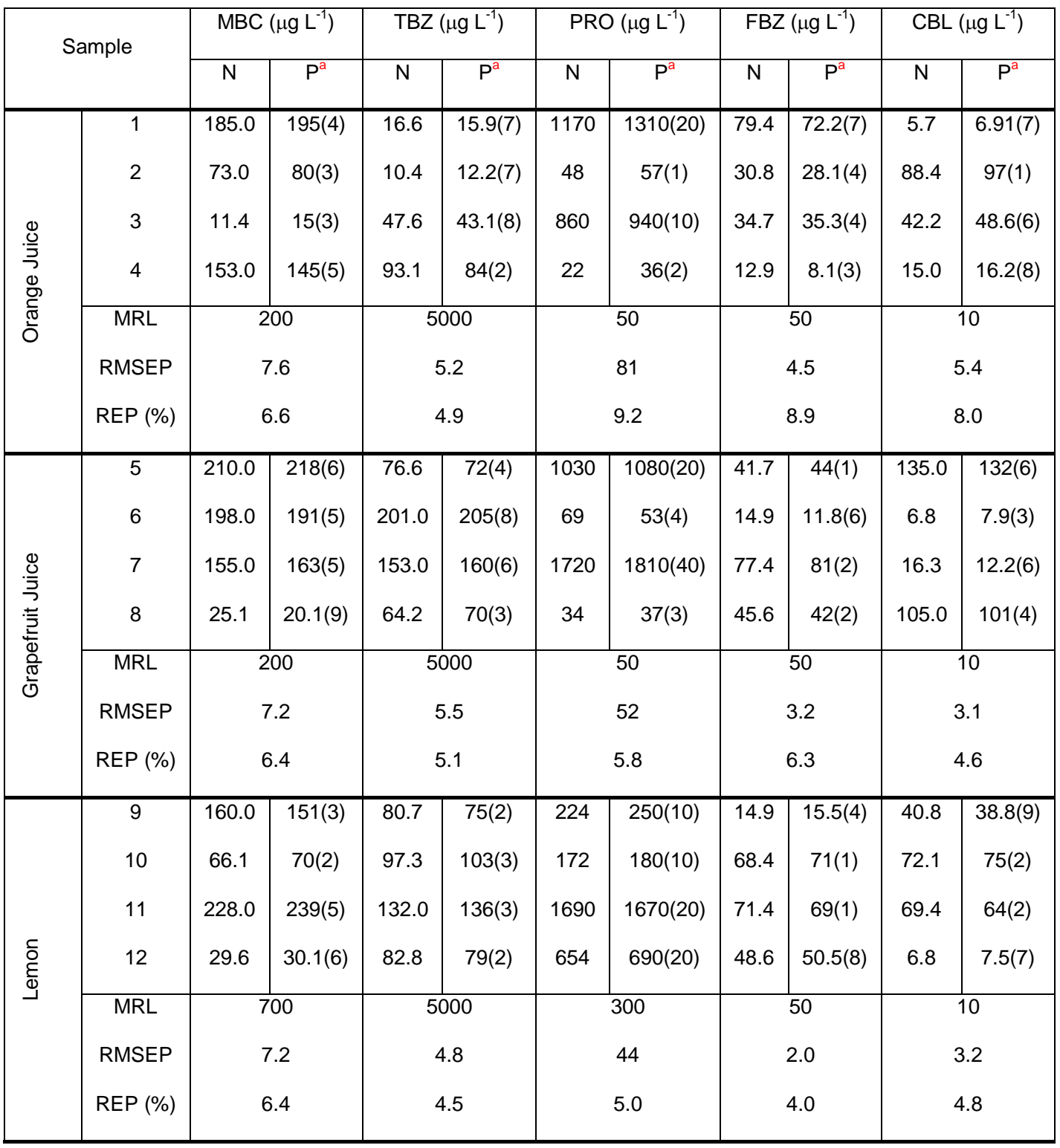

527

$528 \quad$ This table continues in the next page.

529 


\begin{tabular}{|c|c|c|c|c|c|c|c|c|c|c|c|}
\hline \multirow{7}{*}{ 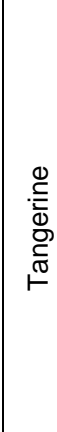 } & 13 & 132.0 & 125(3) & 159.0 & $150(10)$ & 1100 & 1070(20) & 5.9 & $5.2(1)$ & 132.0 & 125(2) \\
\hline & 14 & 198.0 & 204(5) & 97.3 & $102(5)$ & 1200 & $1140(20)$ & 20.8 & $19(1)$ & 15.0 & 12.1(2) \\
\hline & 15 & 93.5 & $97(2)$ & 207.0 & $200(20)$ & 430 & $440(10)$ & 87.3 & $83(1)$ & 80.2 & $78(1)$ \\
\hline & 16 & 59.3 & 64(2) & 132.0 & $126(6)$ & 155 & $125(7)$ & 41.7 & $47(2)$ & 6.8 & $6.6(3)$ \\
\hline & MRL & \multicolumn{2}{|c|}{700} & \multicolumn{2}{|c|}{5000} & \multicolumn{2}{|r|}{300} & \multicolumn{2}{|c|}{50} & \multicolumn{2}{|c|}{10} \\
\hline & RMSEP & \multicolumn{2}{|c|}{5.4} & \multicolumn{2}{|c|}{7.1} & \multicolumn{2}{|r|}{39} & \multicolumn{2}{|c|}{3.6} & \multicolumn{2}{|c|}{3.9} \\
\hline & $\operatorname{REP}(\%)$ & \multicolumn{2}{|c|}{4.8} & \multicolumn{2}{|c|}{6.7} & \multicolumn{2}{|r|}{4.4} & \multicolumn{2}{|c|}{7.0} & \multicolumn{2}{|c|}{5.8} \\
\hline \multirow{7}{*}{ 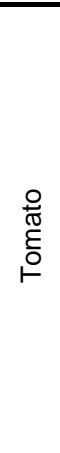 } & 17 & 38.8 & $42(1)$ & 84.9 & $87.6(9)$ & 206 & $\overline{166(4)}$ & 21.8 & 29.6(4) & 124.0 & $131(3)$ \\
\hline & 18 & 80.9 & 76(2) & 97.3 & 91(1) & 1010 & $970(10)$ & 25.8 & $23.5(3)$ & 8.2 & $9.8(2)$ \\
\hline & 19 & 108.0 & $115(2)$ & 128.0 & $121(1)$ & 740 & $797(8)$ & 60.5 & $56.9(7)$ & 59.8 & $65(2)$ \\
\hline & 20 & 213.0 & 203(3) & 15.5 & $21.3(7)$ & 17 & $38(7)$ & 40.0 & $43.6(6)$ & 24.5 & $21(2)$ \\
\hline & MRL & \multicolumn{2}{|c|}{300} & \multicolumn{2}{|c|}{50} & \multicolumn{2}{|r|}{50} & \multicolumn{2}{|r|}{50} & \multicolumn{2}{|c|}{10} \\
\hline & RMSEP & \multicolumn{2}{|c|}{6.8} & \multicolumn{2}{|c|}{5.8} & \multicolumn{2}{|r|}{43} & \multicolumn{2}{|c|}{4.8} & \multicolumn{2}{|c|}{4.8} \\
\hline & REP (\%) & \multicolumn{2}{|c|}{5.9} & \multicolumn{2}{|c|}{5.4} & \multicolumn{2}{|r|}{4.9} & & 9.5 & \multicolumn{2}{|c|}{7.1} \\
\hline & alue & \multicolumn{2}{|c|}{0.389} & \multicolumn{2}{|c|}{0.206} & \multicolumn{2}{|c|}{0.794} & \multicolumn{2}{|c|}{0.439} & \multicolumn{2}{|c|}{0.694} \\
\hline
\end{tabular}

531

$532{ }^{a}$ Standard deviation in parenthesis. $\mathrm{N}=$ nominal, $\mathrm{P}=$ predicted.

533 
535 Figure 1: Chemical structures of the five assayed pesticides.

536

537 Figure 2: Liquid chromatograms ( $\lambda$ of detection: $280 \mathrm{~nm}$ ) for the set of calibration 538 samples. The signal corresponding to each analyte was identified. The subregions 539 selected are highlighted.

540

541 Figure 3: Spectra of pure standards of the five assayed pesticides in medium 542 methanol-water (50:50 v/v). Pesticide concentration: $1 \mathrm{mg} / \mathrm{L}$.

543

544 Figure 4: Results for the analysis of a calibration sample. (A) Surface plot around 545 the first cluster peak (region I) containing the analytes MBC, TBZ, PRO and FBZ 546 (B) MCR-ALS resolved elution profiles for the same sample, with all analytes 547 indicated. (C) Spectral profiles retrieved by MCR-ALS analysis, which are common 548 to all samples. (D) Surface plot around the region II containing CBL (E) MCR-ALS 549 resolved elution profiles in region II. (F) Spectral profiles retrieved by MCR-ALS 550 analysis.

551

552 Figure 5: Results for the analysis of an orange juice sample. (A) Surface plot 553 around the first cluster peak (region I) containing the analytes MBC, TBZ, PRO and 554 FBZ (B) MCR-ALS resolved elution profiles for the same sample, with all analytes 555 indicated. (C) Spectral profiles retrieved by MCR-ALS analysis. (D) Surface plot 
556 around the region II containing CBL (E) MCR-ALS resolved elution profiles in 557 region II. (F) Spectral profiles retrieved by MCR-ALS analysis.

558 

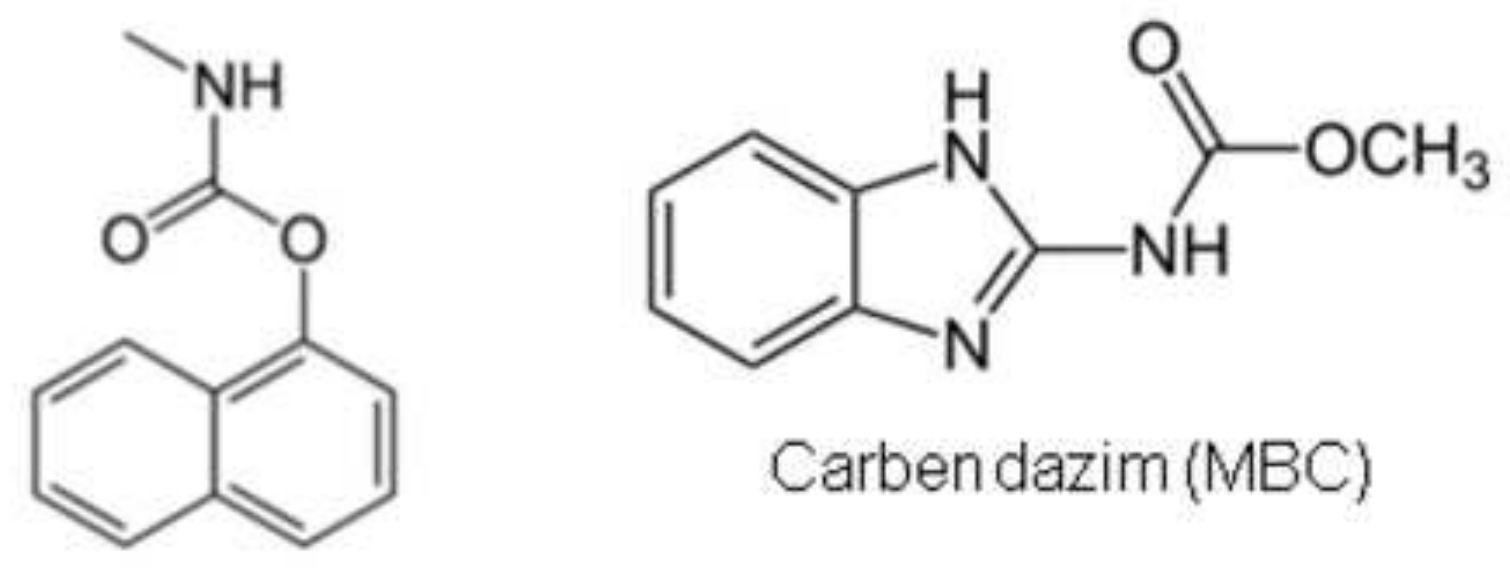

\section{Carben dazim (MBC)}

\section{Carbaryl (CBL)}

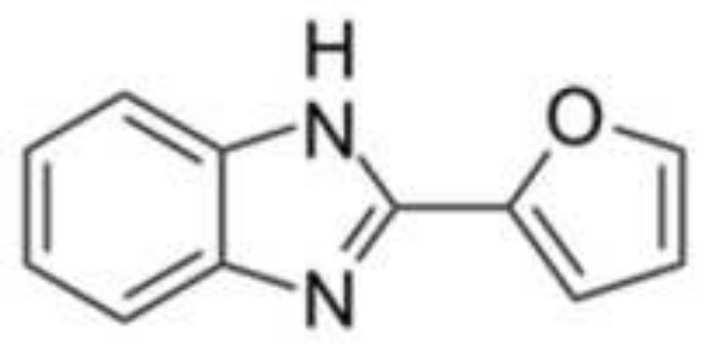

Fuberidazole (FBZ)

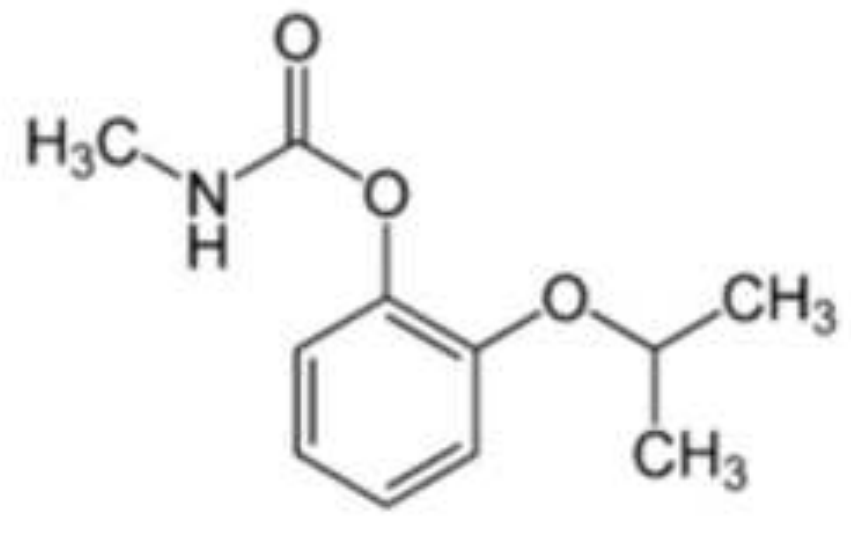

Propoxur (PRO)

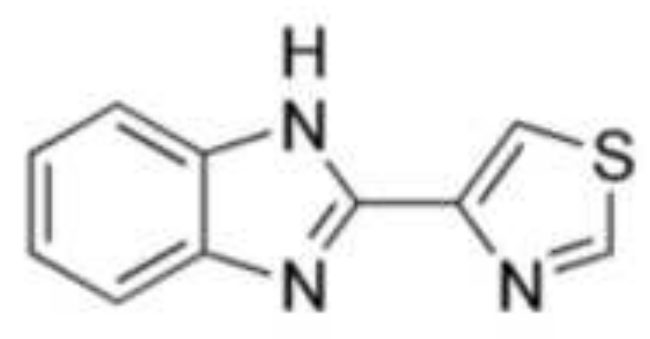

Thiaben dazole (TBZ) 
Figure 2
Click here to download high resolution image

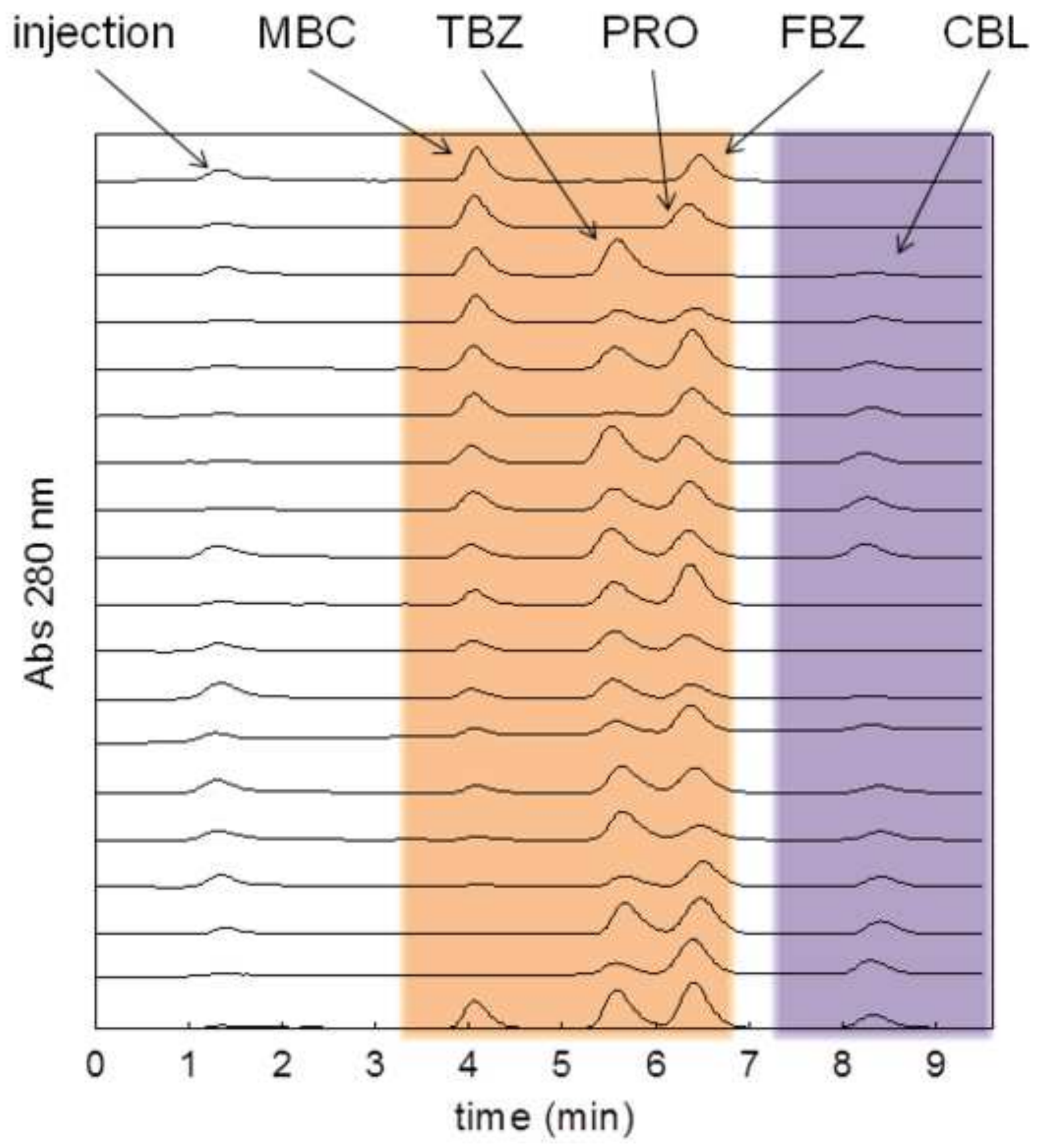




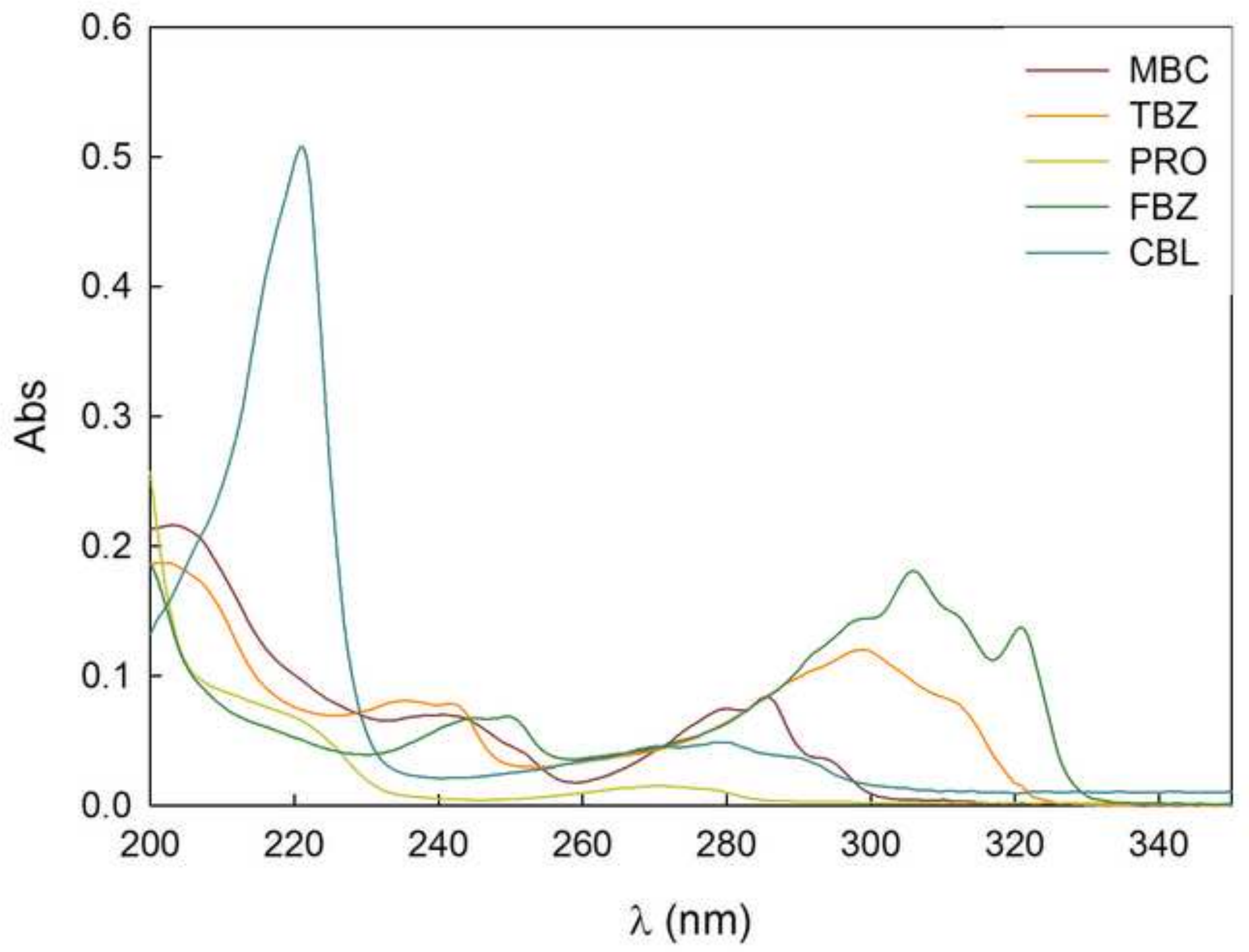



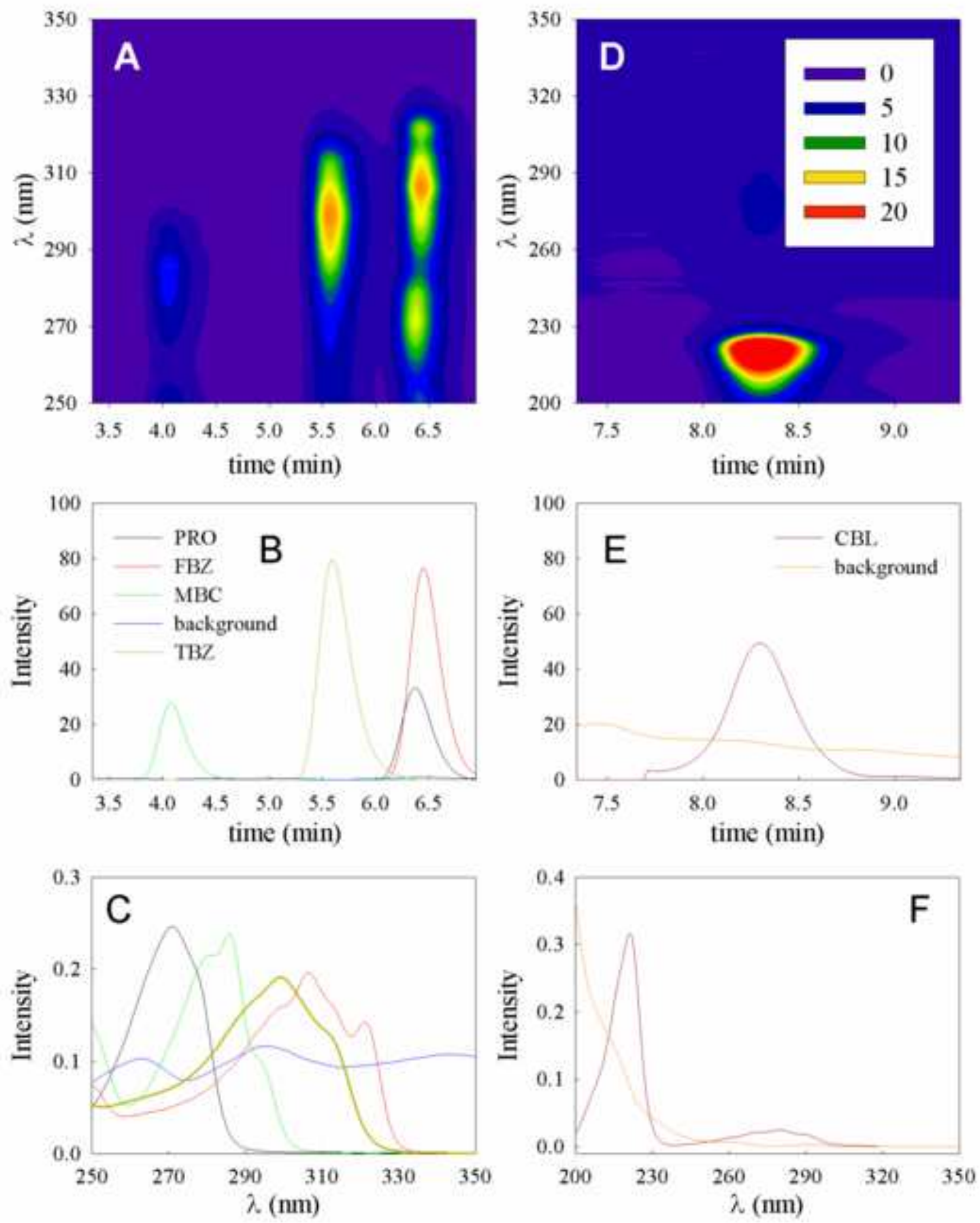

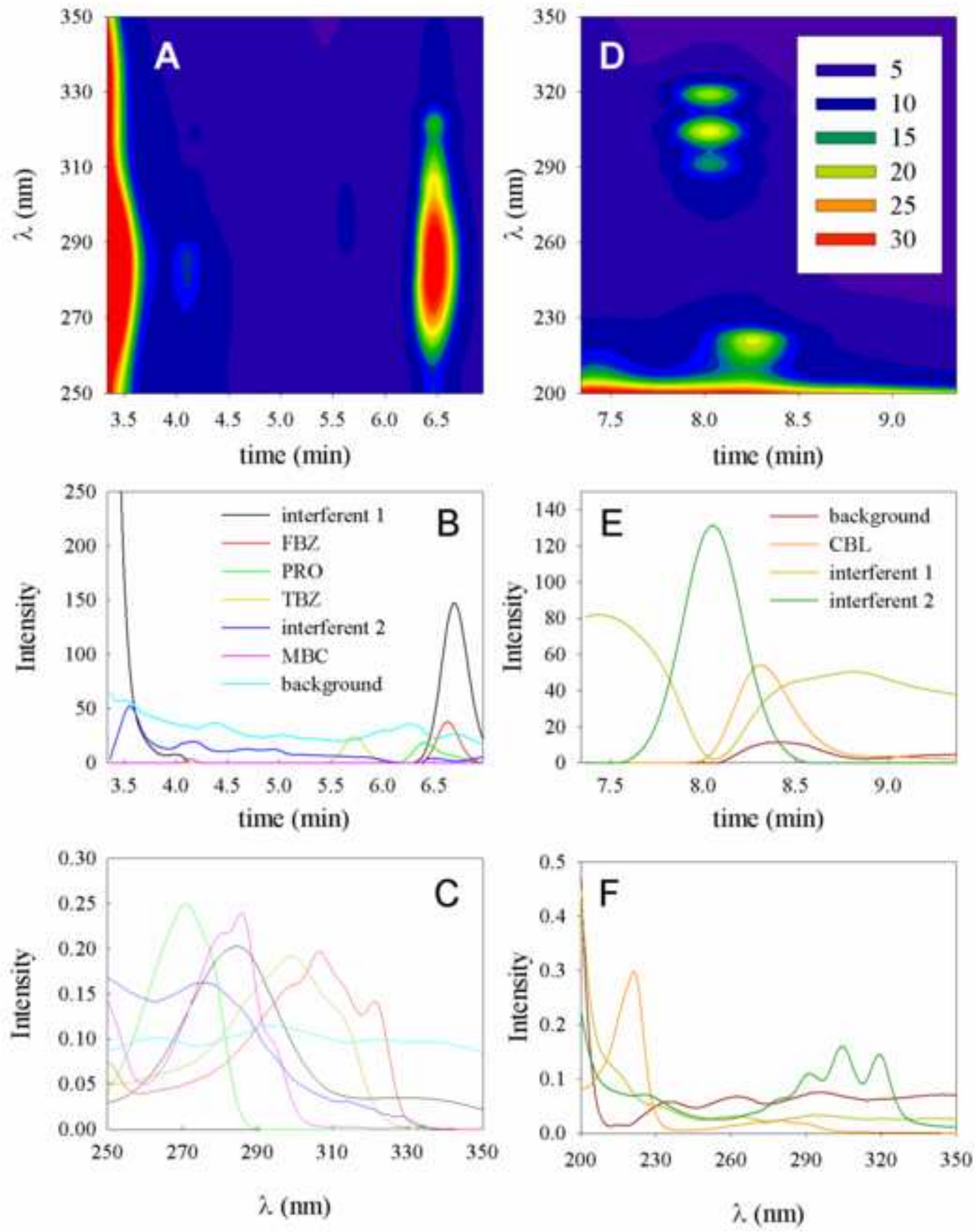